\title{
CliP: subclonal architecture reconstruction of cancer cells in DNA sequencing data using a penalized likelihood model
}

Yujie Jiang ${ }^{1,2, \#}$, Kaixian Yu ${ }^{3, \#}$, Shuangxi Ji1,\#, Seung Jun Shin ${ }^{4}$, Shaolong Cao ${ }^{1}$, Matthew D Montierth ${ }^{1,5}$, Licai Huang ${ }^{3}$, Scott Kopetz ${ }^{6}$, Pavlos Msaouel ${ }^{7}$, Jennifer Rui Wang ${ }^{8}$, Marek Kimmel $^{2}$, Hongtu Zhu ${ }^{9}$, and Wenyi Wang ${ }^{1,3, *}$

${ }^{1}$ Department of Bioinformatics and Computational Biology,

The University of Texas M.D. Anderson Cancer Center, Houston, TX, 77030

${ }^{2}$ Department of Statistics, Rice University, Houston, TX, 77005

${ }^{3}$ Department of Biostatistics,

The University of Texas M.D. Anderson Cancer Center, Houston, TX, 77030

${ }^{4}$ Department of Statistics, Korea University, Seoul, South Korea, 02841

${ }^{5}$ Graduate program in Quantitative Computational Biology, Baylor College of Medicine, Houston, TX, 77030

${ }^{6}$ Department of Gastrointestinal Medical Oncology, The University of Texas M.D. Anderson Cancer Center, Houston, TX, 77030

${ }^{7}$ Department of Genitourinary Medical Oncology, The University of Texas M.D. Anderson Cancer Center, Houston, TX, r7030

${ }^{8}$ Department of Head and Neck Surgery, The University of Texas M.D. Anderson Cancer Center, Houston, TX, 77030

${ }^{9}$ Department of Biostatistics, University of North Carolina at Chapel Hill, Chapel Hill, NC, 27599

$$
\text { \# authors contributed eqally }
$$

*Correspondence: WWang7@mdanderson.org 


\begin{abstract}
Subpopulations of tumor cells characterized by mutation profiles may confer differential fitness and consequently influence prognosis of cancers. Understanding subclonal architecture has the potential to provide biological insight in tumor evolution and advance precision cancer treatment. Recent methods comprehensively integrate single nucleotide variants (SNVs) and copy number aberrations (CNAs) to reconstruct subclonal architecture using whole-genome or whole-exome sequencing (WGS, WES) data from bulk tumor samples. However, the commonly used Bayesian methods require a large amount of computational resources, a prior knowledge of the number of subclones, and extensive post-processing. Regularized likelihood modeling approach, never explored for subclonal reconstruction, can inherently address these drawbacks.

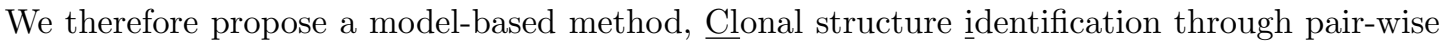
Penalization, or CliP, for clustering subclonal mutations without prior knowledge or postprocessing. The CliP model is applicable to genomic regions with or without CNAs. CliP demonstrates high accuracy in subclonal reconstruction through extensive simulation studies. Utilizing the well-established regularized likelihood framework, CliP takes only 16 hours to process WGS data from 2,778 tumor samples in the ICGC-PCAWG study, and 38 hours to process WES data from 9,564 tumor samples in the TCGA study. In summary, a penalized likelihood framework for subclonal reconstruction will help address intrinsic drawbacks of existing methods and expand the scope of computational analysis for cancer evolution in large cancer genomic studies. The associated software tool is freely available at: https://github.com/wwylab/CliP.
\end{abstract}

Keywords - variant cellular prevalence, intra-tumor heterogeneity, regularized maximum likelihood estimation 


\section{Introduction}

Mutations in tumor cells accumulate from cancer initiation and over the development of cells. Tumor cells generated at different point-in-time, i.e., tumor (sub)clones, may harbor different sets of mutations. These differences form diverse subclonal architecture in individual tumor samples (Nowell 1976; Gerlinger et al. 2012). A tumor clone can be defined as a collection of cells in the tumor sample that harbor the same set of genomic variants, while accordingly, a subclone can be defined as a subpopulation of cells that descend from a clone but further diverge by accumulating additional variants. Thus, a subclone is a subpopulation of tumor cells that have arisen after the most recent common ancestor (MRCA). Mutation profiles may therefore help detect the presence of cancer cell subpopulations (Dentro et al. 2021). Understanding subclonal architecture in bulk tumor samples is crucial for studying biological processes that underlie tumor heterogeneity and evolution, and could mitigate the difficulty in cancer prognosis brought about by complex subclonal composition (Morris et al. 2016; Andor et al. 2016).

Single nucleotide variantion (SNV) is a type of genomic variation that commonly occurs in tumor cells. Following the development of genome sequencing technology, approaches have been developed to call and quantify cancer cell clones through clustering of variant read counts (Dentro et al. 2017). This procedure, called subclonal reconstruction, leverages variant read counts and the associated variant allele fraction (VAF) of somatic mutations, adjusts for copy-number status and tumor purity, in order to identify groups of variants with similar cellular prevalences (CP) (Dentro et al. 2021). These groups of variants are then used to build phylogenetic trees to study a tumor's life history (Deshwar et al. 2015; Jiao et al. 2014; Xiao et al. 2020; Strino et al. 2013; Qiao et al. 2014; Yuan et al. 2015; Malikic et al. 2019).

Many subclonal reconstruction methods have been proposed (Ding et al. 2012; Larson and Fridley 2013; Oesper et al. 2013; Strino et al. 2013; Fischer et al. 2014; Hajirasouliha et al. 2014; Jiao et al. 2014; Miller et al. 2014; Roth et al. 2014; Deshwar et al. 2015) in the past few years. Early subclonal reconstruction methods only worked well on copy number neutral regions. More recent methods showed improved performance by accounting for CNAs with or without SNV allele- 
specific information (Roth et al. 2014; Deshwar et al. 2015; Jiao et al. 2014). These methods address subclonal reconstruction with mixture models, as implemented via Dirichlet processes (DP) or Dirichlet finite mixtures. The computational cost of fully Bayesian methods is high, and grows exponentially with the complexity of the underlying subclonal structure. Alternatively, variational Bayes (Miller et al. 2014) can alleviate the computational burden but still requires prior knowledge on the number of clusters as well as extensive post-processing.

Subclonal reconstruction of SNVs can be considered as finding parsimonious clusters of their underlying subclonal structure. Consequently, it is natural to introduce penalties to induce the sparsity of underlying clusters. Since the seminal LASSO (Tibshirani 1996) was proposed, penalization has been popular for identifying the underlying structure of parameters by pursuing sparsity. As a generalization of sparsity, Ke et al. (2015) introduced homogeneity to infer clustering structures among parameters under which the parameters belonging to the same cluster share an identical structure. The fused LASSO (Tibshirani et al. 2005) that penalizes both parameters and their pairwise differences is an early attempt to seek homogeneity, and Ke et al. (2015) proposed a hybrid penalty as a compromise between the fused LASSO and total variation penalty (Mammen et al. 1997) in order to uncover more complex structure of homogeneity.

In this article, we introduce a subclonal architecture reconstruction method, Clonal structure identification through pair-wise penalization (CliP). We propose to employ a pairwise penalty, a simplified version of the hybrid penalty (Ke et al. 2015) to the likelihood estimation in order to identify subclonal structure. CliP aims to provide substantial computational advantage over existing methods while maintaining a comparable performance in accuracy. This will make CliP a suitable tool for large cancer genomic studies, either by itself or as an add-on to other subclonal reconstruction methods to achieve consensus for most robust outcomes (Dentro et al. 2021). Here we describe the mathematical model and the underlying computational algorithm, a simulation study and application of CliP to the whole-genome sequencing (WGS) data from the International Cancer Genome Consortium Pan Cancer Analysis of Whole Genomes (ICGC-PCAWG), as well as the whole-exome sequencing (WES) data from the Cancer Genome Atlas projects (TCGA). 


\section{Results}

\section{Brief Overview of Subclonal Architecture}

Figure 1a gives an overview of subclonal architecture in a heterogeneous tumor sample, as a result of evolution of cancer cells over time. Cancer cells generate new populations by acquiring oncogenic mutations that allow their descendants to expand relative to the other cell populations. Here, cancer cell population $A$ is divided into two distinct populations ( $B$ and $E$ ) with some cells acquiring an extra copy of a chromosome (population $B$ ) and some cells acquiring additional mutations such as single nucleotide variants (population $E$ ). Similarly, population $B$ gave rise to two distinct cancerous populations, $C$ and $D$. The tumor profile created from testing the sample of Figure 1a could identify up to five cancerous cell populations (A-E) and stromal (normal) cells (F) which are usually assumed to present a diploid genome with no somatic SNVs.

\section{CliP Model}

Our goal is to infer the population structure of heterogeneous tumors based on the measured variant allele fraction (VAF) of their somatic mutations. Distinct from the available tools that utilize Bayesian modeling framework, we aim to identify the subclonal structure through regularization. We first build a binomial model on VAF, adjusting for tumor purity, ploidy, and SNV-specific copy number aberrations at each mutation position; then add an L1 penalty to the likelihood function to impose the homogeneity and sparsity between mutation positions in terms of their cellular prevalence (CP) parameter (see Methods for details). The resulting sparsity structure of SNVs is equivalent to clustering. Therefore, the clustered mutations and their corresponding homogenized $\mathrm{CP}$ values are the output of the CliP model (Figure 1b). Figure 1c,d shows that the number of clusters reduces as the tuning parameter $\lambda$ increases hence the level of sparsity increases in two example cases. 


\section{Simulation Results}

We used simulations to demonstrate that CliP can successfully infer the number of subclones and their fractions, as well as accurately assign SNVs to each cluster (Figure 2a). In general, tumor samples with higher tumor purity and higher read depth present better performaces. Tumor samples with a higher CNA rate or a low purity are more difficult to reconstruct. This trend in performance is consistently observed across methods and for WGS data in particular, is due to the fact that the signal-to-noise ratio becomes too limited with only a few reads assigned to tumor cells or to somatic SNV alleles.

Relative difference in estimated number of clusters ( $r d N C$ ). As is shown in Table 1 and Supplementary Tables 1 and 2, CliP is able to estimate the number of clusters accurately under various scenarios with different purities, CNA rates, and read depths, when the number of clusters $K \leq 3$. Higher number of clusters combined with lower purity leads to undesirable clustering performance, which is expected. At a higher read depth (e.g., $\geq 500 \times$ ) and/or purity (e.g., $\geq$ 0.6), CliP performs well across all scenarios. CliP tends to slightly underestimate the number of clusters as the penalized model combines SNVs with similar CP values rather than assigning them to neighboring clusters. Overall, $\mathrm{CliP}$ presents a reasonable performance in estimating the number of clusters.

Relative difference in clonal fraction $(r d C F)$. Figure 2b and Supplementary Table $\mathbf{3}$ suggest that when there are only two populations (one clonal cluster and one subclonal cluster), CliP can estimate the clonal fraction accurately regardless of read depth, CNA rate, and purity. The clonal fraction estimation is largely affected by the number of clusters (Supplementary Table 4 and 5). When purity is low (at 0.3 ) or total number of clusters is high $(\mathrm{K}=4)$, CliP cannot provide accurate estimate of clonal fractions, which is expected and similar to the performance from other methods. Note the $r d C F$ score will be inflated when the denominator, the true clonal fraction is small, which happens more often when the number of clusters is high. Overall, CliP performs well in recovering the clonal fraction. 
Root Mean Squared Error (RMSE). We use RMSE to assess the recovery of the overall architecture of subclonal composition of SNVs. In general, CliP attains low means and variations in RMSE in most scenarios (Figure 2c, and Supplementary Tables 6-8). RMSE decreases as the read depth increases. In some ideal cases (e.g., the samples with purity of 0.9, depth of 500 or 1000 , and number of clusters at 2), the RMSE is almost 0. RMSE increases as the number of subclones increases, likely due to an increasing chance of incorrect mutation assignment. RMSE does not change significantly over different tumor purity scenarios, supporting the robustness of the CliP results.

Comparison with PhyloWGS. We compared CliP with the popular method PhyloWGS (Deshwar et al. 2015) (see Methods, Figure 2d). Negative values suggest a better performance by CliP. In general, CliP performs comparably to PhyloWGS. It slightly outperforms PhyloWGS when the CNA rate is greater than 0. Further detailed comparisons (Supplementary Figure 1) demonstrate that overall, CliP recovers the clonal fraction better, while presenting similar performances in $r d N C$ and RMSE.

Estimation of purity. CliP can assess tumor purity in tumor samples where CNA events are minimal so that previous CNA-based methods cannot be applied to estimate purity. We conducted a simulation study (see Methods) to evaluate the accuracy of purity estimation (Figure 2e, Figure 2f). Overall, CliP can recover purity with high accuracy, as the majority of relative differences are less than $5 \%$.

\section{Application of CliP to the ICGC-PCAWG and TCGA datasets}

We applied CliP to WGS data from 2,778 PCAWG samples at an average read depth of 30-60× (Dentro et al. 2021). The input somatic SNV and CNA data for CliP were generated using a consensus of 4 mutation callers (The ICGC/TCGA Pan-Cancer Analysis of Whole Genomes Consortium 2020), and 6 copy number calls (Dentro et al. 2021). Overall, it took CliP only 16 hours to finish running all 2,778 PCAWG samples (Figure 3a). We also applied CliP to WES data from 9,564 TCGA samples across 33 cancer types. The input SNV and CNA data were generated as consensus from 5 variant callers and 2 indel callers (Ellrott et al. 2018), and from ASCAT (Van Loo et al. 
2010; Alexandrov et al. 2016), respectively. Our analysis provides a significant update from the previous study (Andor et al. 2016) using PyClone (Roth et al. 2014); we provide results from 9 times more samples and have adjusted for SNV-specific allele-specific CNAs in our model. It took CliP a total of 38 hours to finish running 9,564 TCGA samples (Figure 3b).

\section{Time Complexity}

The computational bottleneck of CliP lies in matrix multiplications, which is optimized by configuring the NumPy module in Python3 with a multi-thread enabled algebra library, e.g. OpenBLAS. No further modification is needed to run CliP with multi-threads, Using 28 cores in parallel can help reduce the runtime, especially for tumor samples with a large amount of SNVs such as those from WGS data (Figure 3a). With 28 cores, CliP can finish running a WGS sample with 5,000 SNVs in 4.1 minutes. Running the same sample using PhyloWGS will take 2,450 minutes. Over selected PCAWG samples with varying total number of SNVs $(100-5,000)$, CliP is at least 200 times faster than PhyloWGS. Both methods were run on the same machine (Intel(R) Xeon(R) Gold 6132 CPU @ 2.60GHz.

To run CliP on 9,564 TCGA samples on the same computer, we randomly divided the samples into 50 batches for parallel analysis, with samples in each batch running in series. We employed only 10 cores for each run. All batches were completed within 38 hours. Figure 3 b shows the computational time required for each TCGA sample. As an example, running a TCGA WES sample with 5,000 SNVs takes about one hour on 10 cores.

\section{Discussion}

In this paper, we present CliP as a novel statistical framework for subclonal reconstruction using penalized likelihood functions. We aim to address intrinsic drawbacks of existing models and better inform cancer evolution. Our modeling framework allows for fast and accurate subclonal structure reconstruction with minimum post-processing procedures. The corresponding software tool is available for download at: https://github.com/wwylab/CliP. 
Under the penalized likelihood framework, we choose SCAD as the penalty function to minimize bias aggregation. All L1-type penalty functions are relaxations of the L0 penalty function. We expect replacing SCAD with the L0 penalty will further improve the performance of CliP. A recent breakthrough on how to solve the L0 penalty (Huang et al. 2018) will be investigated for its application to CliP.

Similar to other available methods, CliP currently takes into consideration only the status of clonal copy number aberrations. CNAs can occur in subclones (Nik-Zainal et al. 2012; JamalHanjani et al. 2014). In rare cases, there may be a subclone where both SNV and subclonal CNAs happen at the same time. CliP will not be able to account for allele-specific copy number correctly for this SNV. However, subclonal CNA calling remains challenging. Its estimates are below the precision level that is required for subclonal reconstruction. In the PCAWG study (The ICGC/TCGA Pan-Cancer Analysis of Whole Genomes Consortium 2020), genomic regions that were suspected to present subclonal CNAs were filtered out from the final CNA call set, which were then used for subclonal reconstruction. Similar considerations were taken in the TCGA study.

Another limitation lies in the computational implementation of matrix multiplications. When the number of SNVs is too large, we downsample the SNVs to ensure the CliP running is within the memory limit. In practice, samples with $<50,000 \mathrm{SNVs}$ can be processed using a 256GB memory without downsampling. Tumor samples in the PCAWG study mostly contains 3,000 to 30,000 SNVs. With the PCAWG data, we designed CliP to run on 2,629 WGS samples with $<50,000$ SNVs, which finished in 8 hours; and run on the remaining 149 samples with > 50,000 SNVs, using multiple rounds of downsampling, which finished also in 8 hours.

Given the fact that CliP introduces an orthogonal statistical framework to address existing drawbacks and executes quickly, it is ultimately suitable for 1) processing large datasets with thousands of samples, and 2) complementing other methods to generate consensus calls. As the size of genomic datasets continues to grow, we believe CliP represents an important step towards fast and accurate subclonal reconstruction. 


\section{Methods}

\section{Model}

In our model, we use $N$ to denote the total number of SNVs in each sample. For each SNV $i$, we use $\left(r_{i}, n_{i}\right)$ to denote the number of reads observed with variant alleles and the total number of reads covering the SNV. We suppose that each sample consists of two populations of cells with respect to the $i$-th SNV: one population contains normal cells or cancer cells that do not present this SNV, and the other population represents cancer cells harboring this SNV. We adopt the infinite site assumption (Kimura 1969): (1) there are an infinite number of sites where mutations can occur, (2) every new mutation occurs at a novel site, and (3) there is no recombination. Under this assumption, there is only one alternative variant at each SNV for each sample, which can vary across different samples. Within cancer cells, SNV $i$ may only occur in one or a few cell populations (Fig 1), therefore we introduce $\beta_{i}$ to denote the cancer cell fraction (CCF) of SNV $i$. With $N$ SNVs, we assume there are $K$ groups of SNVs presenting distinct CCF and $K<<N$.

We take advantage of the observed distribution of VAFs across mutations in heterogeneous tumor samples to build our model. We use $b_{i}^{V}$ to denote the SNV-specific copy number at the $i$-th SNV. This measurement is distinct from allele-specific copy number when the SNV does not occur in all tumor cells. We then use $c_{i}^{N}$ and $c_{i}^{V}$ to denote the total copy number for normal and tumor cell respectively, and $\rho \in[0,1]$ to be the tumor cell proportion, i.e., purity in the sample. We define $\phi_{i}=\rho \beta_{i}$ as the cellular prevalence (CP) of SNV $i$. The SNVs from a common subclone have identical $\mathrm{CP}$ (or $\mathrm{CCF}$ ) and parameter $\phi_{i}$ (or equivalently $\beta_{i}$ ) is of our primary interest.

With the observed data $\left(r_{i}, n_{i}\right)$ at each $\operatorname{SNV} i$, our model assumes $r_{i}$ to follow a binomial distribution $\operatorname{Binom}\left(n_{i}, \theta_{i}\right)$. The expected proportion of the variant allele at the $i$-th $\operatorname{SNV} \theta_{i}$ is further expressed as:

$$
\theta_{i}=f\left(\phi_{i}\right)=\frac{\phi_{i} b_{i}^{V}}{(1-\rho) c_{i}^{N}+\rho c_{i}^{V}}, \quad i=1, \cdots, N
$$




$$
\ell(\phi)=\sum_{i=1}^{N}\left[r_{i} \log f\left(\phi_{i}\right)+\left(n_{i}-r_{i}\right) \log \left(1-f\left(\phi_{i}\right)\right)\right]
$$

The CP, $\phi=\left(\phi_{1}, \cdots, \phi_{N}\right)$ may be estimated by maximizing the corresponding likelihood. However, our primary goal is to identify a homogeneous and sparse structure, i.e., clusters, of the CPs across all SNVs. The penalized estimation is a canonical tool to achieve the homogeneity detection and parameter estimation simultaneously (Hastie et al. 2015). Therefore, we introduce a pair-wise penalty to seek such homogeneity in $\phi$. In order to facilitate the computation, we employ a normal approximation of the binomial random variable, i.e., $\sqrt{n_{i}}\left(r_{i} / n_{i}-\theta_{i}\right) \sim N\left(0, \theta_{i}\left(1-\theta_{i}\right)\right)$. This yields a standard form of penalized objective function with quadratic loss $\tilde{Q}(\phi ; \lambda)$, and the estimator can be obtained by minimizing it over $\phi$, given a tuning parameter $\lambda>0$ that controls the degree of the penalization:

$$
\tilde{Q}(\phi ; \lambda)=\frac{1}{2} \sum_{i=1}^{N} \frac{n_{i}\left(f\left(\phi_{i}\right)-\hat{\theta}_{i}\right)^{2}}{f\left(\phi_{i}\right)\left(1-f\left(\phi_{i}\right)\right)}+\sum_{1 \leq i<j \leq N} p_{\lambda}\left(\left|\phi_{i}-\phi_{j}\right|\right)
$$

where $\hat{\theta}_{i}$ is the estimated proportion of variant allele at the $i$-th SNV, and $p_{\lambda}(\cdot)$ denotes a sparsity pursuing penalty to identify the homogeneity structure in $\phi$, such as LASSO (Tibshirani 1996), SCAD (Fan and Li 2001), and MCP (Zhang et al. 2010) to name a few. Here, we focus on the SCAD penalty that is known to not only enjoy the oracle property but also show improved performance in the model estimation (Fan and Li 2001). Adaptation to other penalty forms will be straightforward.

Input data.

CliP requires information of $c_{i}^{N}, c_{i}^{V}, r_{i}, n_{i}$, and optionally, $\rho$, as its input. Parameters $\rho$ and $c_{i}^{V}$ can be obtained using CNA-based deconvolution methods such as ASCAT (Van Loo et al. 2010) and ABSOLUTE (Carter et al. 2012), whereas $c_{i}^{N}$ is typically set as 2 . In contrast, parameter $b_{i}^{V}$ is not directly observed or estimatable using existing CNA software tools. Following a current 
convention that proved to be effective(Dentro et al. 2017), we assume $b_{i}^{V}$ as:

$$
b_{i}^{V}=\left[\frac{r_{i}}{n_{i}} \frac{1}{\rho}\left(\rho c_{i}^{V}+c_{i}^{N}(1-\rho)\right)\right]_{0}, \quad i=1, \cdots, N
$$

where $[\cdot]_{0}$ rounds $x$ to the nearest positive integer.

Output data for subclonal architecture.

The output for CliP is two-fold: 1) the subclonal structure, i.e., clustering results: cluster number, the total number of SNVs in each cluster, and the estimated CP for each cluster; 2) the mutation assignment, i.e., cluster id for each mutation. This output can then serve as the basis for inference of phylogenetic trees (Dentro et al. 2017).

\section{Computation}

It is not trivial to optimize the objective function in equation (2) because the target variable $\phi$ is bounded, i.e., $\phi=\rho \beta_{i} \in[0,1]$ as both $\beta_{i}$ and $\rho$ are, and the SCAD penalty is not convex. We employ the following re-parametrization of $\phi_{i}$ to mitigate the box constraint:

$$
\omega_{i}=\log \frac{\phi_{i}}{1-\phi_{i}}, \quad i=1, \cdots, N
$$

which yields

$$
\theta_{i}=g\left(\omega_{i}\right)=\frac{b_{i}^{V} e^{\omega_{i}}}{\left(1+e^{\omega_{i}}\right)\left(2-2 \rho+c_{i}^{V} \rho\right)}, \quad i=1, \cdots, N
$$

Note that $\omega_{i}=\omega_{j}$ implies $\phi_{i}=\phi_{j}$, and vice versa, thus the homogeneity pursuit of $\omega^{\prime} s$ is equivalent to that of $\boldsymbol{\phi}$. Now, equation (2) can be equivalently rewritten as a function of $\boldsymbol{\omega}$ :

$$
Q(\boldsymbol{\omega} ; \lambda)=\frac{1}{2} \sum_{i=1}^{N} \frac{n_{i}\left(g\left(\omega_{i}\right)-\hat{\theta}_{i}\right)^{2}}{g\left(\omega_{i}\right)\left(1-g\left(\omega_{i}\right)\right)}+\sum_{1 \leq i<j \leq N} p_{\lambda}\left(\left|\omega_{i}-\omega_{j}\right|\right)
$$

In order to minimize equation (5), we employ the alternating direction method of multipliers 
(ADMM, Boyd et al. 2011), a popular algorithm for large-scale optimization problems. Introducing auxiliary variables $\eta_{i j}=\omega_{i}-\omega_{j}$ for $i<j$, the minimization of (5) is equivalent to the constrained optimization problem,

$$
S(\boldsymbol{\omega}, \boldsymbol{\eta} ; \lambda)=\frac{1}{2} \sum_{i=1}^{N} \frac{n_{i}\left(g\left(\omega_{i}\right)-\hat{\theta}_{i}\right)^{2}}{g\left(\omega_{i}\right)\left(1-g\left(\omega_{i}\right)\right)}+\sum_{1 \leq i<j \leq N} p_{\lambda}\left(\left|\eta_{i j}\right|\right), \quad \text { subject to } \omega_{i}-\omega_{j}-\eta_{i j}=0
$$

By the augmented Lagrangian method, we can change equation (6) into a unconstrained optimization problem, and obtain the estimation of parameters by minimizing

$$
L(\boldsymbol{\omega}, \boldsymbol{\eta}, \boldsymbol{\tau} ; \lambda)=S(\boldsymbol{\omega}, \boldsymbol{\eta} ; \lambda)+\frac{\alpha_{k}}{2} \sum_{i<j}\left(\omega_{i}-\omega_{j}-\eta_{i j}\right)^{2}-\sum_{i<j} \tau_{i j}\left(\omega_{i}-\omega_{j}-\eta_{i j}\right)
$$

where the dual variables $\tau=\left\{\tau_{i j}, i<j\right\}$ are Lagrange multipliers, and $\alpha_{k}$ is the penalty parameter. We compute the estimators of $(\boldsymbol{\omega}, \boldsymbol{\eta}, \boldsymbol{\tau}, \lambda)$ through iterations by the ADMM. Thus, for given $\boldsymbol{\omega}^{(k)}$ and $\boldsymbol{\tau}^{(k)}$ at the $k$-th step, the updates $\boldsymbol{\omega}^{(k+1)}$ and $\boldsymbol{\tau}^{(k+1)}$ are given by

$$
\boldsymbol{\omega}^{(k+1)}=\left(\boldsymbol{B}^{T} \boldsymbol{B}+\alpha_{k} \Delta^{T} \Delta\right)^{-1}\left[\alpha_{k} \Delta(\boldsymbol{\eta}-\boldsymbol{\tau})-\boldsymbol{B}^{T} \boldsymbol{A}\right]
$$

where $\boldsymbol{A}, \boldsymbol{B}$ are matrices associated with $\boldsymbol{\omega}^{(k)}$ (see Supplementary Materials), and $\Delta=\left\{\left(e_{i}-e_{j}\right), i<\right.$ $j\}^{T}$ with $e_{i}$ defined as a $N \times 1$ vector whose $i$-th element is 1 and others are 0 . Then

$$
\boldsymbol{\tau}^{(k+1)}=\boldsymbol{\tau}^{(k)}-\alpha_{k}\left(\Delta \boldsymbol{\omega}^{(k+1)}-\boldsymbol{\eta}^{(k+1)}\right)
$$

The update of $\eta_{i j}$ at the $(k+1)$-th iteration is obtained by the formula

$$
\hat{\eta}_{i j}= \begin{cases}\operatorname{ST}\left(\delta_{i j}, \lambda / \alpha_{k}\right) & \text { if }\left|\delta_{i j}\right| \leq \lambda+\lambda / \alpha_{k} \\ \frac{S T\left(\delta_{i j}, \gamma \lambda /\left((\gamma-1) \alpha_{k}\right)\right)}{1-1 /\left((\gamma-1) \alpha_{k}\right)} & \text { if } \lambda+\lambda / \alpha_{k}<\left|\delta_{i j}\right| \leq \gamma \lambda \\ \delta_{i j} & \text { if }\left|\delta_{i j}\right|>\gamma \lambda,\end{cases}
$$

where $S T(x, t)$ is the soft thresholding operator defined as $\operatorname{sign}(x)(|x|-t)]$ if $|x| \geq t$ and 0 otherwise. 
Finally, the ADMM algorithm to solve the proposed model is summarized as follows: 1) Initialize the estimates. Normally, a random initialization works well. We initialize $\omega_{i}^{(0)}=\log \frac{r_{i} / n_{i}}{1-\left(r_{i} / n_{i}\right)}$, $\eta_{i j}^{(0)}=\omega_{i}^{(0)}-\omega_{j}^{(0)}$, and $\boldsymbol{\tau}^{(0)}=\mathbf{1}_{N}$, where $\mathbf{1}_{N}$ is defined as a vector of 1 's with length $N .2$ ) At iteration $m+1$, compute $\left(\boldsymbol{\omega}^{(\boldsymbol{m}+\mathbf{1})}, \boldsymbol{\eta}^{(\boldsymbol{m + 1})}, \boldsymbol{\tau}^{(\boldsymbol{m + 1})}\right)$ as described above. 3) Terminate the algorithm if the stopping criterion (e.g., $\left.\max \left(\eta_{i j}^{(m+1)}-\eta_{i j}^{(m)}\right)<0.01\right)$ is met at step $m+1$. If not, go back to step 2 and repeat. Further details of our algorithm can be found in Supplementary Materials.

\section{Post-processing}

Given the fact that there is a hyper-parameter $\lambda$ in our model, its selection is important for finding a balance between over- and under-fitting. The size of $\lambda$ controls the number of clusters. In general, higher $\lambda$ values return fewer clusters. We introduce a selection approach, which focuses on the match of clonal mutations, rather than a perfect match of the overall structure. The automated $\lambda$ selection pipeline is as follows: 1) For each sample, run CliP with 11 different $\lambda$ : 0.01, 0.03, 0.05, $0.075,0.1,0.125,0.15,0.175,0.2,0.225,0.25$ (figure 1 c \& d). 2) For each sample, compute a score: $A=\left|\frac{\max (C P)-\text { purity }}{\text { purity }}\right|$. If there are one or more results that satisfy $A<0.05$, we select the largest $\lambda$ associated with those results. If all scores $A$ are greater than 0.05 , we choose the $\lambda$ associated with the smallest $A$.

Theoretically, it is not necessary to perform further manual post-processing because $\phi_{i}^{\prime} \mathrm{s}$ naturally define the subclonal architecture. But in practice, due to sequencing error and over-dispersion in some samples (especially samples with fewer than $50 \mathrm{SNVs}$ ), there may be small clusters with a negligible amount of SNVs $(<5$ SNVs or $<1 \%$ of total SNVs for instance). In such cases, we merge the cluster with its closest neighbor cluster (smallest distance between centers).

\section{Simulation Study}

We conducted simulation studies to demonstrate how CliP improves computation efficiency without sacrificing performance. Simulated data are generated as follows (see Figure 2a). We first set purity $\rho$ as $0.3,0.6$, or 0.9 . We then set $K$, the number of clusters of SNVs with unique CCF, 
as 2,3 , or 4 . The CCF for the $k$ th cluster of SNVs, denoted by $\beta_{k}$, is sampled from Uniform $(0.2,1)$ with minimum distance $d=0.2$ between any two clusters. We then have CP $\phi_{k}=\rho \beta_{k}$ for the $k$ th cluster. The number of SNVs in each cluster is simulated from Uniform(200,500). To generate copy number status at each SNV, we take the following steps:

1. Initialize the status of mutated/reference allele of SNV at $1 / 1$.

2. Sample altering status of SNV (1, 0 for with or without CNA, respectively) from Bernoulli $(p)$ with different values of $p \in\{0,0.1,0.2\}$.

3. For each SNV with CNA, sample the numbers of copies of mutated allele with SNV, mutated allele without SNV, and reference allele from a discrete uniform distribution over $\{0,1,2,3,4,5\}$

For each SNV $i$, we sampled the total number of reads $n_{i} \sim \operatorname{Poissson}(N)$, where $\mathrm{N}$ is the average read depth for the sample, taking values values $N \in\{100,500,1,000\}$. We then sampled the variant reads $r_{i}$ from $\operatorname{Binomial}\left(n_{i} ; \theta_{i}\right)$, where $\theta_{i}$ is calculated from the purity, CP and SNV-specific copy number using equation (1). We generated 50 samples for each combination of read depth, purity, CNA rate, and number of clusters. This gives us a total of $50 \times 81=4050$ samples.

\section{Evaluation Metrics}

We evaluated how well CliP recovers the subclonal architecture using three metrics that measure bias in estimated number of clusters, clonal fraction, and CP across all variants, respectively. First we calculated the relative difference in number of clusters $r d N C=\frac{\left|N_{e}-N_{t}\right|}{N_{t}}$, where $N_{e}$ is the estimated number of clusters and $N_{t}$ the true number of clusters. We then calculated the relative difference in clonal fraction $r d C F=\frac{\left|C_{e}-C_{t}\right|}{C_{t}}$, where $C_{e}$ and $C_{t}$ represent the CliP estimated clonal fraction and the truth clonal fraction, respectively. We further calculated a root mean squared error $R M S E=\sqrt{\frac{1}{N} \sum_{n=1}^{N}\left(\frac{\hat{\phi}_{i}-\phi_{i}}{\rho}\right)^{2}}$, where $N$ is the total number of SNVs, $\hat{\phi}_{i}$ is the estimated CP, $\phi_{i}$ is the true $\mathrm{CP}$ for $\mathrm{SNV} i$, and $\rho$ is the true purity of the sample which was used to standardize the dynamic ranges of different samples. In all these metrics, smaller values indicate better performance, and 0 indicates a correct reconstruction. 
For a cross-method comparison, a mean score is calculated as the mean of the aforementioned three scores for each sample. We introduce a normalized difference score between CliP and PhyloWGS, which is calculated as $\frac{2\left(m_{c}-m_{p}\right)}{m_{c}+m_{p}}$, where $m_{c}$ and $m_{p}$ are scaled scores for CliP and PhyloWGS respectively. A negative normalized difference score suggests better performance in CliP.

\section{Purity For Copy Number Alteration Silent Samples}

In general, purity can be recovered from CNA regions of the sample, which is the basis for most subclonal reconstruction methods. However, some tumors may present limited or no CNAs, e.g., those from thyroid or prostate cancer. CliP may be used to estimate purity under these scenarios. In such a case, the estimation of SNV specific copy numbers $\left(b_{i}^{V}\right.$ 's) is not needed; they will be 1 at the heterozygous sites and 2 at the homozygous sites. Assuming the cancer cell fraction (CCF) of clonal mutations is 1 (i.e. $\max _{i}\left(\beta_{i}\right)=1$ ), CliP can directly estimate the purity $(\rho)$.

Simulation study for purity estimation. We set the number of clusters of SNVs with unique CCF values $K$ at 2,3 , or 4 . For each $\mathrm{SNV} i$, we sampled the total number of reads $n_{i} \sim \operatorname{Pois}(N)$, where $N=60,200,500$, or 1000 . We generated 100 samples for each combination of read depth and number of clusters, where the purity value $\rho_{0}$ of each sample is sampled from a uniform distribution Unif $(0.3,1)$. Relative difference of purity change is used to measure the accuracy of purity estimation, which is defined as $\left(\rho_{e}-\rho_{0}\right) / \rho_{0}$, where $\rho_{e}$ and $\rho_{0}$ denote the estimated and true purity, respectively.

\section{PCAWG and TCGA datasets (The ICGC/TCGA Pan-Cancer Analysis of Whole} Genomes Consortium 2020; Dentro et al. 2021)

Our study is built on the International Cancer Genome Consortium's Pan-Cancer Analysis of Whole Genomes (PCAWG) initiative, which represents the largest dataset of cancer whole-genome sequences to date. In total, 2,778 cancer samples from 2,658 distinct donors were included in the final dataset, comprising 2,605 primary tumors and 173 metastases or recurrences. In comparison to whole exome sequencing, whole-genome sequencing data provides 1 - 2 orders of magnitude more point mutations, greater resolution to detect CNAs, and the ability to call structural variants 
(SVs). These advantages greatly increase the breadth and depth of our subclonal analyses. The PCAWG working group in tumor heterogeneity and evolution applied an ensemble of six state-ofthe-art copy number callers and 11 subclonal reconstruction methods, and developed approaches to integrate their calls into a high-confidence consensus (Dentro et al. 2021). Consensus copy number calls were assigned 'tiers' based on the level of agreement between different callers. Consensus copy number profiles, SNVs, and purity estimates served as input to 11 subclonal SNV-clustering methods, which included CliP.

We further include whole-exome sequencing data from the Cancer Genome Atlas (TCGA) as a complement of our real data analysis. The TCGA data is the world's largest and richest collection of genomic data, molecularly characterizing primary cancer and matched normal samples across 33 cancer types. A total of 10,039 TCGA patient samples with matched somatic mutation calls from WES and copy number segments and tumor purity from ASCAT (Van Loo et al. 2010) were processed using the CliP pipeline. The somatic mutation calls are the consensus set from 7 variant calling methods (including Indelocator (Banerji et al. 2012), MuSE (Fan et al. 2016), MuTect (Cibulskis et al. 2013), Pindel (Ye et al. 2009), RADIA (Radenbaugh et al. 2014), SomaticSniper (Larson et al. 2012), and VarScan (Koboldt et al. 2012)) and downloaded from Ellrott et al. (Ellrott et al. 2018). ASCAT tumor purity and ploidy data were downloaded from Alexandrov et al. (Alexandrov et al. 2016). After removing samples with $<10 \mathrm{SNVs}$, subclonal reconstruction of a total of 9,564 samples was generated.

\section{References}

Alexandrov, L. B., Ju, Y. S., Haase, K., Van Loo, P., Martincorena, I., Nik-Zainal, S., Totoki, Y., Fujimoto, A., Nakagawa, H., Shibata, T., Campbell, P. J., Vineis, P., Phillips, D. H. and Stratton, M. R. (2016). Mutational signatures associated with tobacco smoking in human cancer, Science 354(6312): 618-622.

URL: http://science.sciencemag.org/

Andor, N., Graham, T. A., Jansen, M., Xia, L. C., Aktipis, C. A., Petritsch, C., Ji, H. P. and Maley, 
C. C. (2016). Pan-cancer analysis of the extent and consequences of intratumor heterogeneity, Nature Medicine 22(1): 105-113.

URL: https://www.nature.com/articles/nm.3984

Banerji, S., Cibulskis, K., Rangel-Escareno, C., Brown, K. K., Carter, S. L., Frederick, A. M., Lawrence, M. S., Sivachenko, A. Y., Sougnez, C., Zou, L. et al. (2012). Sequence analysis of mutations and translocations across breast cancer subtypes, Nature 486(7403): 405-409.

URL: https://www.nature.com/articles/nature11154

Boyd, S., Parikh, N., Chu, E., Peleato, B. and Eckstein, J. (2011). Distributed optimization and statistical learning via the alternating direction method of multipliers, Found. Trends Mach. Learn. 3(1): 1-122.

URL: https://web.stanford.edu/boyd/papers/pdf/admm_distr_stats.pdf

Carter, S. L., Cibulskis, K., Helman, E., McKenna, A., Shen, H., Zack, T., Laird, P. W., Onofrio, R. C., Winckler, W., Weir, B. A. et al. (2012). Absolute quantification of somatic dna alterations in human cancer, Nature biotechnology 30(5): 413-421.

URL: https://www.nature.com/articles/nbt.2203

Cibulskis, K., Lawrence, M. S., Carter, S. L., Sivachenko, A., Jaffe, D., Sougnez, C., Gabriel, S., Meyerson, M., Lander, E. S. and Getz, G. (2013). Sensitive detection of somatic point mutations in impure and heterogeneous cancer samples, Nature Biotechnology 31(3): 213-219.

URL: https://www.nature.com/articles/nbt.2514

Dentro, S. C., Leshchiner, I., Haase, K., Tarabichi, M., Wintersinger, J., Deshwar, A. G., Yu, K., Rubanova, Y., Macintyre, G., Vázquez-García, I. et al. (2021). Portraits of genetic intra-tumour heterogeneity and subclonal selection across cancer types, Cell (In Press) .

URL: https://www.biorxiv.org/content/10.1101/312041v4

Dentro, S. C., Wedge, D. C. and Van Loo, P. (2017). Principles of reconstructing the subclonal architecture of cancers, Cold Spring Harbor perspectives in medicine 7(8): a026625.

URL: https://www.ncbi.nlm.nih.gov/pmc/articles/PMC5538405/ 
Deshwar, A. G., Vembu, S., Yung, C. K., Jang, G. H., Stein, L. and Morris, Q. (2015). Phylowgs: reconstructing subclonal composition and evolution from whole-genome sequencing of tumors, Genome Biol 16: 35.

URL: https://www.ncbi.nlm.nih.gov/pubmed/25786235

Ding, L., Ley, T. J., Larson, D. E., Miller, C. A., Koboldt, D. C. and Welch, J. S. (2012). Clonal evolution in relapsed acute myeloid leukaemia revealed by whole-genome sequencing, Nature 481(7382): 506-510.

URL: https://doi.org/10.1038/nature10738

Ellrott, K., Bailey, M. H., Saksena, G., Covington, K. R., Kandoth, C., Stewart, C., Hess, J., Ma, S., Chiotti, K. E., McLellan, M. D., Sofia, H. J. et al. (2018). Scalable Open Science Approach for Mutation Calling of Tumor Exomes Using Multiple Genomic Pipelines, Cell Systems 6(3): 271281.e7.

URL: https://www.ncbi.nlm.nih.gov/pmc/articles/PMC6075717/

Fan, J. and Li, R. (2001). Variable selection via nonconcave penalized likelihood and its oracle properties, Journal of the American statistical Association 96(456): 1348-1360.

URL: https://www.tandfonline.com/doi/abs/10.1198/016214501753382273

Fan, Y., Xi, L., Hughes, D. S. T., Zhang, J., Zhang, J., Futreal, P. A., Wheeler, D. A. and Wang, W. (2016). MuSE: accounting for tumor heterogeneity using a sample-specific error model improves sensitivity and specificity in mutation calling from sequencing data, Genome biology 17(1): 178 . URL: https://genomebiology.biomedcentral.com/articles/10.1186/s13059-016-1029-6

Fischer, A., Vázquez-García, I., Illingworth, C. J. and Mustonen, V. (2014). High-definition reconstruction of clonal composition in cancer, Cell Reports 7(5): 1740-1752.

URL: https://doi.org/10.1016/j.celrep.2014.04.055

Gerlinger, M. et al. (2012). Intratumor heterogeneity and branched evolution revealed by multiregion sequencing, New England Journal of Medicine 366(10): 883-892.

URL: http://www.nejm.org/doi/full/10.1056/NEJMoa1113205 
Hajirasouliha, I., Mahmoody, A. and Raphael, B. J. (2014). A combinatorial approach for analyzing intra-tumor heterogeneity from high-throughput sequencing data, Bioinformatics 30(12): i78-i86. URL: https://doi.org/10.1093/bioinformatics/btu284

Hastie, T., Tibshirani, R. and Wainwright, M. (2015). Statistical learning with sparsity: the lasso and generalizations, CRC press.

URL: https://web.stanford.edu/ hastie/StatLearnSparsity/

Huang, J., Jiao, Y., Liu, Y. and Lu, X. (2018). A constructive approach to 10 penalized regression, The Journal of Machine Learning Research 19(1): 403-439.

URL: https://jmlr.org/papers/volume19/17-194/17-194.pdf

Jamal-Hanjani, M. et al. (2014). Tracking genomic cancer evolution for precision medicine: the lung tracerx study, PLoS Biol 12(7): e1001906.

URL: https://journals.plos.org/plosbiology/article?id=10.1371/journal.pbio.1001906

Jiao, W., Vembu, S., Deshwar, A. G., Stein, L. and Morris, Q. (2014). Inferring clonal evolution of tumors from single nucleotide somatic mutations, BMC bioinformatics 15(1): 1-16.

URL: https://bmcbioinformatics.biomedcentral.com/articles/10.1186/1471-2105-15-35

Ke, Z. T., Fan, J. and Wu, Y. (2015). Homogeneity pursuit, Journal of the American Statistical Association 110(509): 175-194.

URL: https://www.tandfonline.com/doi/abs/10.1080/01621459.2014.892882?journalCode=uasa20

Kimura, M. (1969). The number of heterozygous nucleotide sites maintained in a finite population due to steady flux of mutations, Genetics 61(4): 893.

URL: https://www.genetics.org/content/61/4/893

Koboldt, D. C., Zhang, Q., Larson, D. E., Shen, D., McLellan, M. D., Lin, L., Miller, C. A., Mardis, E. R., Ding, L. and Wilson, R. K. (2012). VarScan 2: Somatic mutation and copy number alteration discovery in cancer by exome sequencing, Genome Research 22(3): 568-576.

URL: http://www.genome.org/cgi/doi/10.1101/gr.129684.111. 
Larson, D. E., Harris, C. C., Chen, K., Koboldt, D. C., Abbott, T. E., Dooling, D. J., Ley, T. J., Mardis, E. R., Wilson, R. K. and Ding, L. (2012). SomaticSniper: identification of somatic point mutations in whole genome sequencing data, Bioinformatics 28(3): 311-317.

URL: https://academic.oup.com/bioinformatics/article-lookup/doi/10.1093/bioinformatics/btr665

Larson, N. B. and Fridley, B. L. (2013). Purbayes: estimating tumor cellularity and subclonality in next-generation sequencing data, Bioinformatics 29(15): 1888-1889.

URL: https://doi.org/10.1093/bioinformatics/btt293

Malikic, S., Jahn, K., Kuipers, J., Sahinalp, S. C. and Beerenwinkel, N. (2019). Integrative inference of subclonal tumour evolution from single-cell and bulk sequencing data, Nature communications 10(1): 1-12.

URL: https://www.nature.com/articles/s41467-019-10737-5

Mammen, E., van de Geer, S. et al. (1997). Locally adaptive regression splines, The Annals of Statistics 25(1): 387-413.

URL: $\quad$ https://projecteuclid.org/journals/annals-of-statistics/volume-25/issue-1/Locallyadaptive-regression-splines/10.1214/aos/1034276635.full

Miller, C. A., White, B. S., Dees, N. D., Griffith, M., Welch, J. S. and Griffith, O. L. (2014). Sciclone: inferring clonal architecture and tracking the spatial and temporal patterns of tumor evolution, PLoS Comput Biol 10(8): e1003665.

URL: https://doi.org/10.1371/journal.pcbi.1003665

Morris, L. G., Riaz, N., Desrichard, A., Senbabaoglu, Y., Hakimi, A. A., Makarov, V., Reis-Filho, J. S. and Chan, T. A. (2016). Pan-cancer analysis of intratumor heterogeneity as a prognostic determinant of survival, Oncotarget 7(9): 10051-63.

URL: https://www.oncotarget.com/article/7067/text/

Nik-Zainal, S., Van Loo, P., Wedge, D. C., Alexandrov, L. B., Greenman, C. D., Lau, K. W., Raine, K., Jones, D., Marshall, J., Ramakrishna, M. et al. (2012). The life history of 21 breast cancers, 
Cell 149(5): 994-1007.

URL: https://www.cell.com/fulltext/S0092-8674(12)00527-2

Nowell, P. C. (1976). The clonal evolution of tumor cell populations, Science 194(4260): 23-28.

URL: https://science.sciencemag.org/content/194/4260/23.long

Oesper, L., Mahmoody, A. and Raphael, B. (2013). Theta: inferring intra-tumor heterogeneity from high-throughput dna sequencing data, Genome Biol 14: 1-21.

URL: https://doi.org/10.1186/gb-2013-14-7-r80

Qiao, Y., Quinlan, A. R., Jazaeri, A. A., Verhaak, R. G., Wheeler, D. A. and Marth, G. T. (2014). Subcloneseeker: a computational framework for reconstructing tumor clone structure for cancer variant interpretation and prioritization, Genome biology 15(8): 1-15.

URL: https://genomebiology.biomedcentral.com/articles/10.1186/s13059-014-0443-x

Radenbaugh, A. J., Ma, S., Ewing, A., Stuart, J. M., Collisson, E. A., Zhu, J. and Haussler, D. (2014). RADIA: RNA and DNA Integrated Analysis for Somatic Mutation Detection, PLoS ONE 9(11): e111516.

URL: https://dx.plos.org/10.1371/journal.pone.0111516

Roth, A., Khattra, J., Yap, D., Wan, A., Laks, E. and Biele, J. (2014). Pyclone: statistical inference of clonal population structure in cancer, Nat Methods 11: 396-398.

URL: https://doi.org/10.1038/nmeth.2883

Strino, F., Parisi, F., Micsinai, M. and Kluger, Y. (2013). Trap: a tree approach for fingerprinting subclonal tumor composition, Nucleic acids research 41(17): e165-e165.

URL: https://academic.oup.com/nar/article/41/17/e165/2411666

The ICGC/TCGA Pan-Cancer Analysis of Whole Genomes Consortium (2020). Pan-cancer analysis of whole genomes, Nature 578(7793): 82-93.

URL: https://www.nature.com/articles/s41586-020-1969-6 
Tibshirani, R. (1996). Regression shrinkage and selection via the lasso, Journal of the Royal Statistical Society: Series B (Methodological) 58(1): 267-288.

URL: http://statweb.stanford.edu/tibs/ftp/lasso-retro.pdf

Tibshirani, R., Saunders, M., Rosset, S., Zhu, J. and Knight, K. (2005). Sparsity and smoothness via the fused lasso, Journal of the Royal Statistical Society: Series B (Statistical Methodology) 67(1): $91-108$.

URL: https://web.stanford.edu/group/SOL/papers/fused-lasso-JRSSB.pdf

Van Loo, P., Nordgard, S. H., Lingjærde, O. C., Russnes, H. G., Rye, I. H., Sun, W., Weigman, V. J., Marynen, P., Zetterberg, A., Naume, B. et al. (2010). Allele-specific copy number analysis of tumors, Proceedings of the National Academy of Sciences 107(39): 16910-16915.

URL: https://www.pnas.org/content/107/39/16910.long

Xiao, Y., Wang, X., Zhang, H., Ulintz, P. J., Li, H. and Guan, Y. (2020). Fastclone is a probabilistic tool for deconvoluting tumor heterogeneity in bulk-sequencing samples, Nature communications 11(1): 1-11.

URL: https://www.nature.com/articles/s41467-020-18169-2

Ye, K., Schulz, M. H., Long, Q., Apweiler, R. and Ning, Z. (2009). Pindel: a pattern growth approach to detect break points of large deletions and medium sized insertions from paired-end short reads, Bioinformatics 25(21): 2865-2871.

URL: https://academic.oup.com/bioinformatics/article-lookup/doi/10.1093/bioinformatics/btp394

Yuan, K., Sakoparnig, T., Markowetz, F. and Beerenwinkel, N. (2015). Bitphylogeny: a probabilistic framework for reconstructing intra-tumor phylogenies, Genome biology 16(1): 1-16.

URL: https://genomebiology.biomedcentral.com/articles/10.1186/s13059-015-0592-6

Zhang, C.-H. et al. (2010). Nearly unbiased variable selection under minimax concave penalty, The Annals of statistics 38(2): 894-942.

URL: $\quad$ https://projecteuclid.org/journals/annals-of-statistics/volume-38/issue-2/Nearlyunbiased-variable-selection-under-minimax-concave-penalty/10.1214/09-AOS729.full 


\section{Figures and Tables}

\begin{tabular}{|c|c|c|c|c|}
\hline \multirow{2}{*}{ Purity } & \multirow{2}{*}{ CNA rate } & \multicolumn{3}{|c|}{ Read Depth } \\
\cline { 3 - 5 } & & 100 & 500 & 1000 \\
\hline \multirow{3}{*}{0.3} & 0 & $0.01(0.071)$ & $0(0)$ & $0(0)$ \\
\cline { 2 - 5 } & 0.1 & $0.01(0.071)$ & $0(0)$ & $0(0)$ \\
\cline { 2 - 5 } & 0.2 & $0(0)$ & $0(0)$ & $0(0)$ \\
\hline \hline \multirow{3}{*}{0.6} & 0 & $0.06(0.164)$ & $0(0)$ & $0(0)$ \\
\cline { 2 - 5 } & 0.1 & $0.07(0.202)$ & $0(0)$ & $0(0)$ \\
\cline { 2 - 5 } & 0.2 & $0.03(0.120)$ & $0(0)$ & $0(0)$ \\
\hline \hline \multirow{3}{*}{0.9} & 0 & $0.01(0.071)$ & $0(0)$ & $0(0)$ \\
\cline { 2 - 5 } & 0.1 & $0.02(0.099)$ & $0(0)$ & $0(0)$ \\
\cline { 2 - 5 } & 0.2 & $0.05(0.182)$ & $0(0)$ & $0(0)$ \\
\hline
\end{tabular}

Table 1: The mean and standard deviation of $r d N C$ s for CliP at $K=2$. 

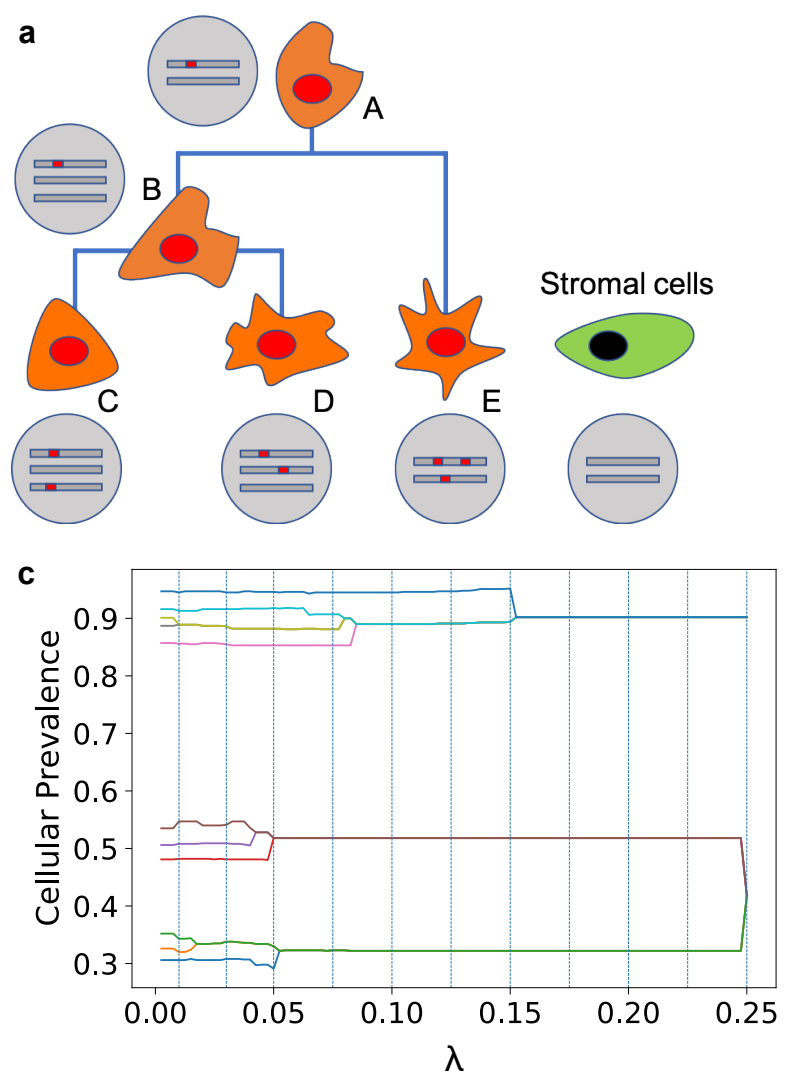
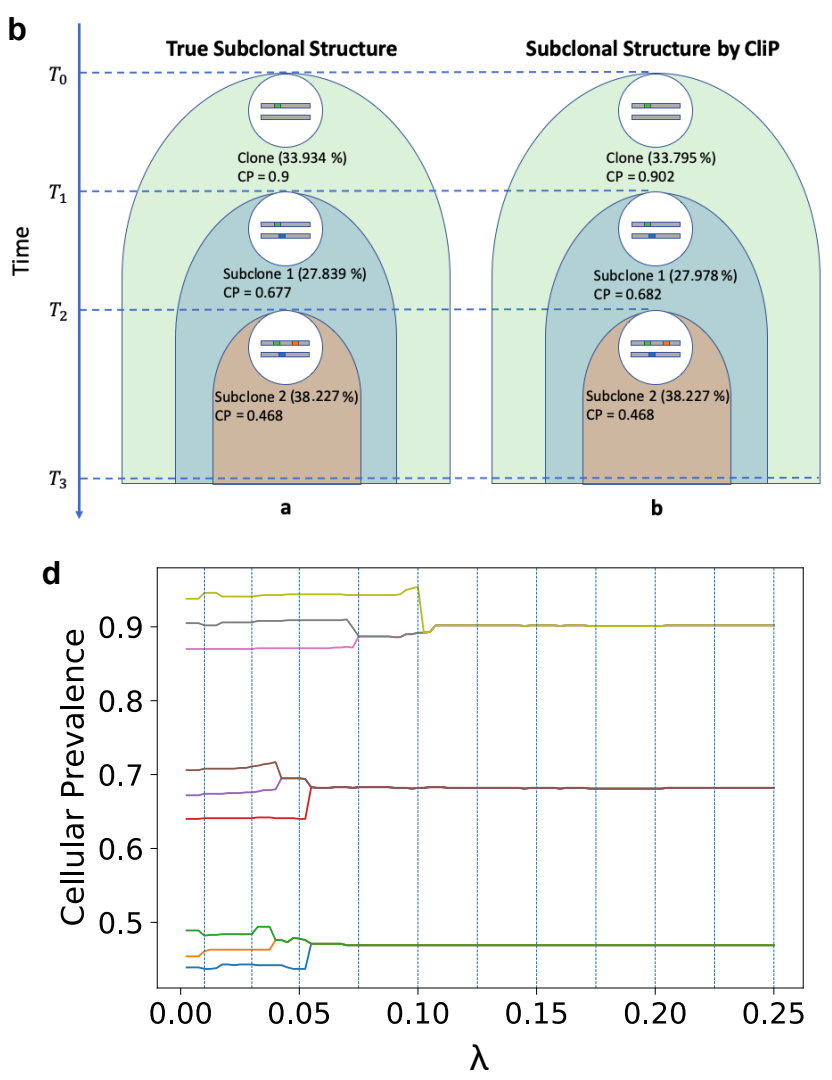

Figure 1: Development of intratumor heterogeneity and subclonal reconstruction in cancer cells. a. Overview of subclonal architecture in a tumor sample. Each circle contains the genotype of the corresponding cancer cell population at the time of sampling. b. Illustration of cancer cell composition over time as well as the resulting distribution of variant allele fractions (VAFs). The subclonal structure is quantified as the homogeneous cellular prevalence (CP) estimation for clusters of SNVs. We simulated sequencing data for a tumor sample with read depth at 1,000 , tumor purity at 0.9 , number of clusters at 3 , and CNA rate at 0 . Starting from the tumor initiation time $T_{0}$, there is one group of SNVs in all cancer cells. These cells then continue to evolve by acquiring more mutations. Suppose we sample these cells at time points $T_{1}$ and $T_{2}$, we would observe new groups of cancer cells with a distinct set of SNVs. This tumor is finally sampled at $T_{3}$. Each circle contains the genotype of a representative SNV in the newly generated cancerous cells by $T_{3}$, while the three circles together demonstrate an evolutionary trajectory of the corresponding cancerous cells. The somatic SNVs that distinguish new cancerous cells from parental cells should form clusters and are colored in green, blue and brown, respectively. b(i) shows the true subclonal structure, with the corresponding $\mathrm{CP}$ values and proportions of cancer cell populations. b(ii) shows the subclonal structure inferred by CliP. $\mathbf{c} \& \mathbf{d}$. CliP infers clusters of SNVs and their corresponding $\mathrm{CP}$ values using a penalty function, which varies over the tuning parameter $\lambda$. We run $\mathrm{CliP}$ with 11 values of $\lambda(0.01,0.03,0.05,0.075,0.1,0.125,0.15,0.175,0.2,0.225,0.25)$, represented by 11 vertical dotted lines. The CPs shown in this example are estimated using $100 \lambda$ values evenly spaced between 0.01 and 0.25 . Between $\lambda$ values, the nearest $\mathrm{CP}$ estimates are connected, showing paths of convergence, hence clustering, in the $\mathrm{CP}$ estimation by CliP. 

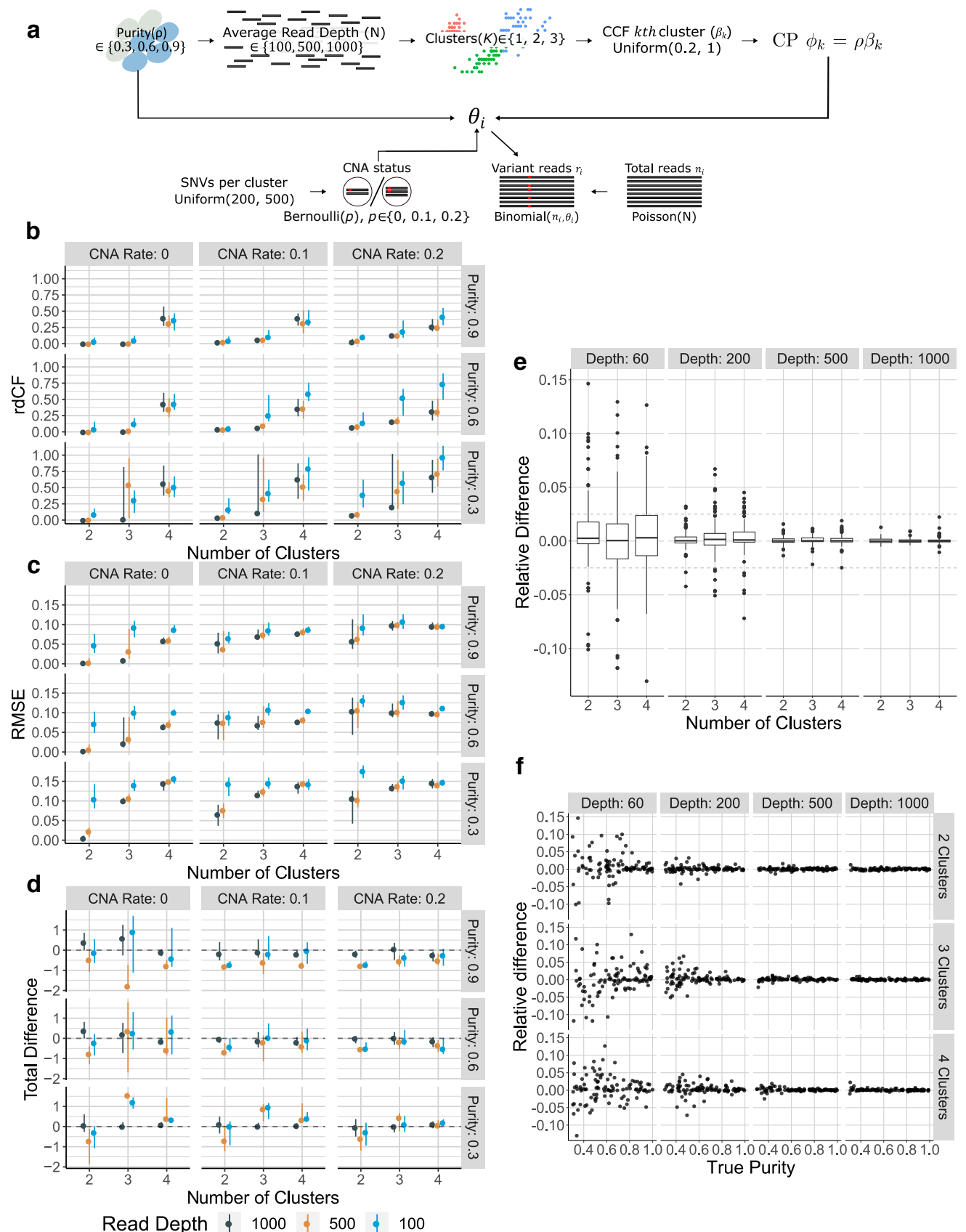

Figure 2: Simulation design and results. a. Overview of the simulation study. We ran CliP with simulated samples of different purity values $(0.3,0.6,0.9)$, different numbers of clusters $(2$, $3,4)$, different CNA rates $(0,0.1,0.2)$, and different read depths $(100,500,1000)$, a total of 81 combinations). b - d. We use the "ball-stick" plots to demonstrate the performance of CliP, where the ball indicates the median value, and upper and lower reach of each stick indicate the 25th and 75th percentiles, respectively. b\&c. $r d C F$ and $R M S E$ scores for all simulated scenarios. d. Normalized overall score differences between CliP and PhyloWGS under these scenarios. e\&f Performance of CliP in purity estimation under various simulation scenarios. This simulation is performed separately from the above model evaluation. 
a

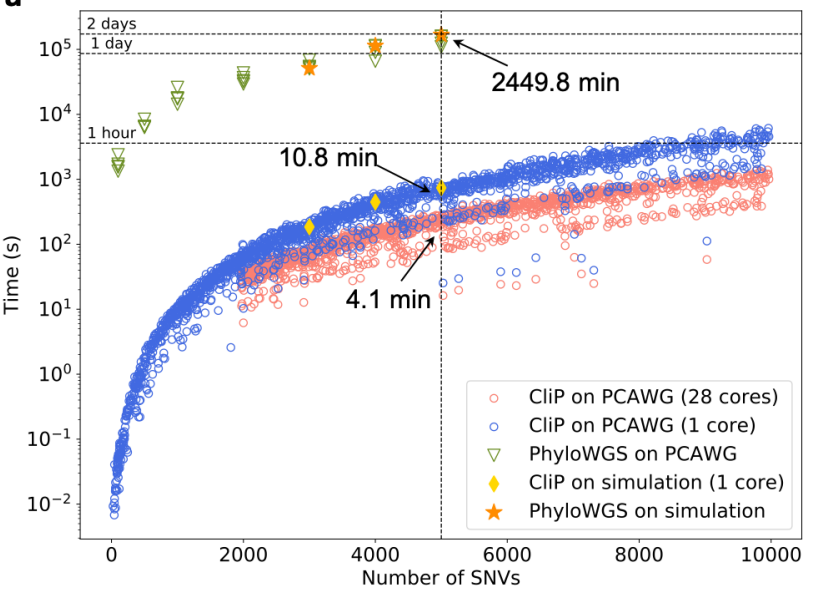

b

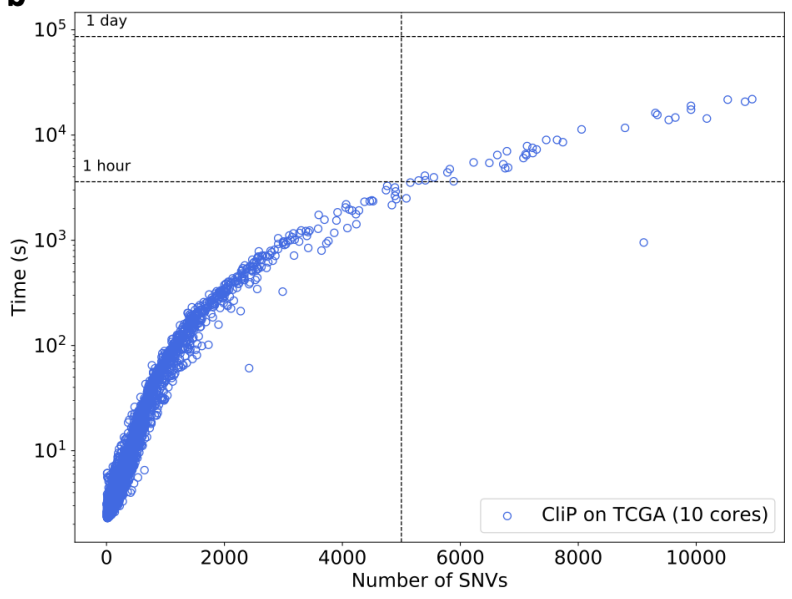

Figure 3: Computational time for subclonal reconstruction on the PCAWG and TCGA datasets. a, Comparison of computational time needed by CliP (single- or multi-core) and PhyloWGS. b, Computational time needed by CliP on the TCGA dataset. In both $\mathbf{a}$, and $\mathbf{b}$,, the $\mathrm{y}$ axis is the running time in seconds, at a $\log 10$ scale. We highlight the median running time for CliP ( 1 core \& 28 cores for PCAWG and 10 cores for TCGA) and PhyloWGS at number of SNVs $=5,000$. 


\section{CliP: subclonal architecture reconstruction of cancer cells in DNA sequencing data using a penalized likelihood model}

Yujie Jiang ${ }^{1,2, \#}$, Kaixian $\mathrm{Yu}^{3, \#}$, Shuangxi Ji1,\#, Seung Jun Shin ${ }^{4}$, Shaolong Cao ${ }^{1}$, Matthew D Montierth ${ }^{1,5}$, Licai Huang ${ }^{3}$, Scott Kopetz ${ }^{6}$, Pavlos Msaouel ${ }^{7}$, Jennifer Rui Wang ${ }^{8}$, Marek Kimmel $^{2}$, Hongtu Zhu ${ }^{9}$, and Wenyi Wang ${ }^{1,3, *}$

${ }^{1}$ Department of Bioinformatics and Computational Biology,

The University of Texas M.D. Anderson Cancer Center, Houston, TX, 77030

${ }^{2}$ Department of Statistics, Rice University, Houston, TX, 77005

${ }^{3}$ Department of Biostatistics,

The University of Texas M.D. Anderson Cancer Center, Houston, TX, 77030

${ }^{4}$ Department of Statistics, Korea University, Seoul, South Korea, 02841

${ }^{5}$ Graduate program in Quantitative Computational Biology, Baylor College of Medicine, Houston, TX, 77030

${ }^{6}$ Department of Gastrointestinal Medical Oncology, The University of Texas M.D. Anderson Cancer Center, Houston, TX, 77030

${ }^{7}$ Department of Genitourinary Medical Oncology, The University of Texas M.D. Anderson Cancer Center, Houston, TX, 77030

${ }^{8}$ Department of Head and Neck Surgery, The University of Texas M.D. Anderson Cancer Center, Houston, $T X, 77030$

${ }^{9}$ Department of Biostatistics, University of North Carolina at Chapel Hill, Chapel Hill, NC, 27599

\# authors contributed eqally

*Correspondence: WWang7@mdanderson.org

\section{Contents}

1 Supplementary Figures and Tables 2

2 Supplementary Text 6

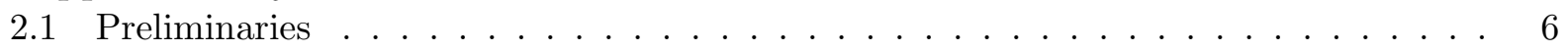

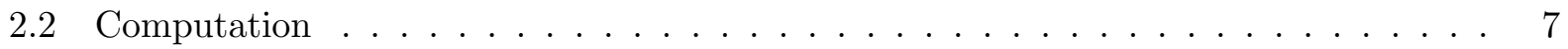

3 Supplementary Induction $r$ 


\section{Supplementary Figures and Tables}

\begin{tabular}{|c|c|c|c|c|}
\hline \multirow{2}{*}{ Purity } & \multirow{2}{*}{ CNA rate } & \multicolumn{3}{|c|}{ Read Depth } \\
\cline { 3 - 5 } & & 100 & 500 & 1000 \\
\hline \multirow{3}{*}{0.3} & 0 & $0.313(0.080)$ & $0.327(0.047)$ & $0.313(0.080)$ \\
\cline { 2 - 5 } & 0.1 & $0.300(0.101)$ & $0.327(0.047)$ & $0.333(0)$ \\
\cline { 2 - 5 } & 0.2 & $0.313(0.080)$ & $0.320(0.066)$ & $0.327(0.047)$ \\
\hline \hline \multirow{3}{*}{0.6} & 0 & $0.187(0.180)$ & $0.093(0.151)$ & $0.127(0.163)$ \\
\cline { 2 - 5 } & 0.1 & $0.200(0.165)$ & $0.140(0.166)$ & $0.087(0.148)$ \\
\cline { 2 - 5 } & 0.2 & $0.227(0.157)$ & $0.113(0.160)$ & $0.107(0.157)$ \\
\hline \multirow{3}{*}{0.9} & 0 & $0.267(0.178)$ & $0.120(0.162)$ & $0.053(0.123)$ \\
\cline { 2 - 5 } & 0.1 & $0.253(0.239)$ & $0.126(0.163)$ & $0.067(0.135)$ \\
\cline { 2 - 5 } & 0.2 & $0.220(0.198)$ & $0.087(0.148)$ & $0.047(0.117)$ \\
\hline
\end{tabular}

Supplementary Table 1: The means and standard deviations of $r d N C$ for CliP at $K=3$

\begin{tabular}{|c|c|c|c|c|}
\hline \multirow{2}{*}{ Purity } & \multirow{2}{*}{ CNA rate } & \multicolumn{3}{|c|}{ Read Depth } \\
\cline { 3 - 5 } & & 100 & 500 & 1000 \\
\hline \multirow{3}{*}{0.3} & 0 & $0.475(0.076)$ & $0.495(0.035)$ & $0.495(0.035)$ \\
\cline { 2 - 5 } & 0.1 & $0.470(0.082)$ & $0.480(0.069)$ & $0.470(0.082)$ \\
\cline { 2 - 5 } & 0.2 & $0.495(0.035)$ & $0.490(0.049)$ & $0.480(0.069)$ \\
\hline \hline \multirow{3}{*}{0.6} & 0 & $0.090(0.121)$ & $0.075(0.136)$ & $0.060(0.119)$ \\
\cline { 2 - 5 } & 0.1 & $0.150(0.152)$ & $0.065(0.122)$ & $0.015(0.060)$ \\
\cline { 2 - 5 } & 0.2 & $0.115(0.145)$ & $0.050(0.101)$ & $0.045(0.120)$ \\
\hline \multirow{3}{*}{0.9} & 0 & $0.260(0.159)$ & $0(0)$ & $0.010(0.071)$ \\
\cline { 2 - 5 } & 0.1 & $0.235(0.155)$ & $0.050(0.101)$ & $0.010(0.049)$ \\
\cline { 2 - 5 } & 0.2 & $0.180(0.168)$ & $0.075(0.116)$ & $0.070(0.113)$ \\
\hline
\end{tabular}

Supplementary Table 2: The means and standard deviations of $r d N C$ for CliP at $K=4$

\begin{tabular}{|c|c|c|c|c|}
\hline \multirow{2}{*}{ Purity } & \multirow{2}{*}{ CNA rate } & \multicolumn{3}{|c|}{ Read Depth } \\
\cline { 2 - 5 } & & 100 & 500 & 1000 \\
\hline \multirow{3}{*}{0.3} & 0 & $0.187(0.240)$ & $0.010(0.014)$ & $0.003(0.006)$ \\
\cline { 2 - 5 } & 0.1 & $0.259(0.227)$ & $0.045(0.019)$ & $0.039(0.022)$ \\
\cline { 2 - 5 } & 0.2 & $0.413(0.239)$ & $0.089(0.037)$ & $0.070(0.041)$ \\
\hline \hline \multirow{3}{*}{0.6} & 0 & $0.119(0.182)$ & $0.006(0.012)$ & $0.000(0.003)$ \\
\cline { 2 - 5 } & 0.1 & $0.100(0.143)$ & $0.038(0.022)$ & $0.036(0.022)$ \\
\cline { 2 - 5 } & 0.2 & $0.242(0.210)$ & $0.075(0.037)$ & $0.064(0.041)$ \\
\hline \multirow{3}{*}{0.9} & 0 & $0.061(0.071)$ & $0.012(0.028)$ & $0.001(0.005)$ \\
\cline { 2 - 5 } & 0.1 & $0.081(0.090)$ & $0.026(0.018)$ & $0.024(0.017)$ \\
\cline { 2 - 5 } & 0.2 & $0.121(0.092)$ & $0.044(0.028)$ & $0.040(0.036)$ \\
\hline
\end{tabular}

Supplementary Table 3: The means and standard deviations of $r d C F$ for CliP at $K=2$ 


\begin{tabular}{|c|c|c|c|c|}
\hline \multirow{2}{*}{ Purity } & \multirow{2}{*}{ CNA rate } & \multicolumn{3}{|c|}{ Read Depth } \\
\cline { 2 - 5 } & & 100 & 500 & 1000 \\
\hline \multirow{3}{*}{0.3} & 0 & $0.343(0.224)$ & $0.528(0.474)$ & $0.328(0.494)$ \\
\cline { 2 - 5 } & 0.1 & $0.461(0.262)$ & $0.532(0.449)$ & $0.499(0.514)$ \\
\cline { 2 - 5 } & 0.2 & $0.510(0.324)$ & $0.578(0.426)$ & $0.530(0.454)$ \\
\hline \hline \multirow{3}{*}{0.6} & 0 & $0.173(0.165)$ & $0.041(0.059)$ & $0.030(0.085)$ \\
\cline { 2 - 5 } & 0.1 & $0.342(0.250)$ & $0.097(0.051)$ & $0.066(0.026)$ \\
\cline { 2 - 5 } & 0.2 & $0.486(0.284)$ & $0.196(0.135)$ & $0.169(0.103)$ \\
\hline \hline \multirow{3}{*}{0.9} & 0 & $0.117(0.146)$ & $0.034(0.079)$ & $0.003(0.005)$ \\
\cline { 2 - 5 } & 0.1 & $0.170(0.163)$ & $0.061(0.025)$ & $0.072(0.065)$ \\
\cline { 2 - 5 } & 0.2 & $0.234(0.180)$ & $0.127(0.039)$ & $0.131(0.035)$ \\
\hline
\end{tabular}

Supplementary Table 4: The means and standard deviations of $r d C F$ for CliP at $K=3$

\begin{tabular}{|c|c|c|c|c|}
\hline \multirow{2}{*}{ Purity } & \multirow{2}{*}{ CNA rate } & \multicolumn{3}{|c|}{ Read Depth } \\
\cline { 3 - 5 } & & 100 & 500 & 1000 \\
\hline \multirow{3}{*}{0.3} & 0 & $0.515(0.204)$ & $0.500(0.267)$ & $0.603(0.301)$ \\
\cline { 2 - 5 } & 0.1 & $0.715(0.303)$ & $0.551(0.311)$ & $0.622(0.327)$ \\
\cline { 2 - 5 } & 0.2 & $0.947(0.267)$ & $0.729(0.301)$ & $0.661(0.306)$ \\
\hline \hline \multirow{3}{*}{0.6} & 0 & $0.452(0.183)$ & $0.374(0.219)$ & $0.463(0.216)$ \\
\cline { 2 - 5 } & 0.1 & $0.599(0.191)$ & $0.341(0.165)$ & $0.368(0.178)$ \\
\cline { 2 - 5 } & 0.2 & $0.710(0.269)$ & $0.408(0.243)$ & $0.372(0.247)$ \\
\hline \multirow{3}{*}{0.9} & 0 & $0.337(0.173)$ & $0.345(0.146)$ & $0.429(0.195)$ \\
\cline { 2 - 5 } & 0.1 & $0.404(0.187)$ & $0.342(0.215)$ & $0.389(0.171)$ \\
\cline { 2 - 5 } & 0.2 & $0.422(0.196)$ & $0.299(0.143)$ & $0.316(0.196)$ \\
\hline
\end{tabular}

Supplementary Table 5: The means and standard deviations of $r d C F$ for CliP at $K=4$

\begin{tabular}{|c|c|c|c|c|}
\hline \multirow{2}{*}{ Purity } & \multirow{2}{*}{ CNA rate } & \multicolumn{3}{|c|}{ Read Depth } \\
\cline { 3 - 5 } & & 100 & 500 & 1000 \\
\hline \multirow{3}{*}{0.3} & 0 & $0.108(0.037)$ & $0.022(0.013)$ & $0.008(0.008)$ \\
\cline { 2 - 5 } & 0.1 & $0.139(0.034)$ & $0.072(0.026)$ & $0.062(0.032)$ \\
\cline { 2 - 5 } & 0.2 & $0.172(0.029)$ & $0.102(0.036)$ & $0.090(0.044)$ \\
\hline \hline \multirow{3}{*}{0.6} & 0 & $0.074(0.036)$ & $0.012(0.011)$ & $0.003(0.003)$ \\
\cline { 2 - 5 } & 0.1 & $0.086(0.028)$ & $0.068(0.036)$ & $0.068(0.035)$ \\
\cline { 2 - 5 } 0.9 & 0.2 & $0.127(0.024)$ & $0.097(0.043)$ & $0.093(0.048)$ \\
\hline \multirow{3}{*}{0} & 0 & $0.050(0.031)$ & $0.011(0.016)$ & $0.005(0.009)$ \\
\cline { 2 - 5 } & 0.1 & $0.066(0.023)$ & $0.053(0.031)$ & $0.055(0.032)$ \\
\cline { 2 - 5 } & 0.2 & $0.100(0.038)$ & $0.075(0.034)$ & $0.076(0.045)$ \\
\hline
\end{tabular}

Supplementary Table 6: The means and standard deviations of RMSE for CliP at $K=2$ 


\begin{tabular}{|c|c|c|c|c|}
\hline \multirow{2}{*}{ Purity } & \multirow{2}{*}{ CNA rate } & \multicolumn{3}{|c|}{ Read Depth } \\
\cline { 3 - 5 } & & 100 & 500 & 1000 \\
\hline \multirow{3}{*}{0.3} & 0 & $0.140(0.023)$ & $0.107(0.017)$ & $0.099(0.023)$ \\
\cline { 2 - 5 } & 0.1 & $0.145(0.019)$ & $0.122(0.017)$ & $0.117(0.013)$ \\
\cline { 2 - 5 } & 0.2 & $0.149(0.025)$ & $0.135(0.020)$ & $0.134(0.015)$ \\
\hline \hline \multirow{3}{*}{0.6} & 0 & $0.100(0.027)$ & $0.048(0.035)$ & $0.048(0.041)$ \\
\cline { 2 - 5 } & 0.1 & $0.110(0.022)$ & $0.087(0.029)$ & $0.076(0.028)$ \\
\cline { 2 - 5 } 0.9 & 0.2 & $0.127(0.030)$ & $0.109(0.026)$ & $0.106(0.025)$ \\
\hline \multirow{3}{*}{0.1} & $0.091(0.029)$ & $0.048(0.039)$ & $0.019(0.030)$ \\
\cline { 2 - 5 } & 0.1 & $0.090(0.023)$ & $0.079(0.022)$ & $0.074(0.018)$ \\
\cline { 2 - 5 } & $0.107(0.027)$ & $0.100(0.017)$ & $0.097(0.015)$ \\
\hline
\end{tabular}

Supplementary Table 7: The means and standard deviations of RMSE for CliP at $K=3$

\begin{tabular}{|c|c|c|c|c|}
\hline \multirow{2}{*}{ Purity } & \multirow{2}{*}{ CNA rate } & \multicolumn{3}{|c|}{ Read Depth } \\
\cline { 3 - 5 } & & 100 & 500 & 1000 \\
\hline \multirow{3}{*}{0.3} & 0 & $0.153(0.015)$ & $0.147(0.014)$ & $0.137(0.018)$ \\
\cline { 2 - 5 } & 0.1 & $0.142(0.017)$ & $0.140(0.017)$ & $0.133(0.020)$ \\
\cline { 2 - 5 } & 0.2 & $0.146(0.011)$ & $0.140(0.012)$ & $0.143(0.015)$ \\
\hline \hline \multirow{3}{*}{0.6} & 0 & $0.101(0.014)$ & $0.071(0.024)$ & $0.068(0.017)$ \\
\cline { 2 - 5 } & 0.1 & $0.104(0.012)$ & $0.081(0.016)$ & $0.078(0.010)$ \\
\cline { 2 - 5 } & 0.2 & $0.113(0.019)$ & $0.098(0.010)$ & $0.100(0.014)$ \\
\hline \multirow{3}{*}{0.9} & 0 & $0.099(0.031)$ & $0.062(0.012)$ & $0.063(0.014)$ \\
\cline { 2 - 5 } & 0.1 & $0.091(0.020)$ & $0.081(0.011)$ & $0.077(0.007)$ \\
\cline { 2 - 5 } & 0.2 & $0.098(0.022)$ & $0.097(0.011)$ & $0.098(0.011)$ \\
\hline
\end{tabular}

Supplementary Table 8: The mean and standard deviation of RMSE for CliP at $K=4$ 
a

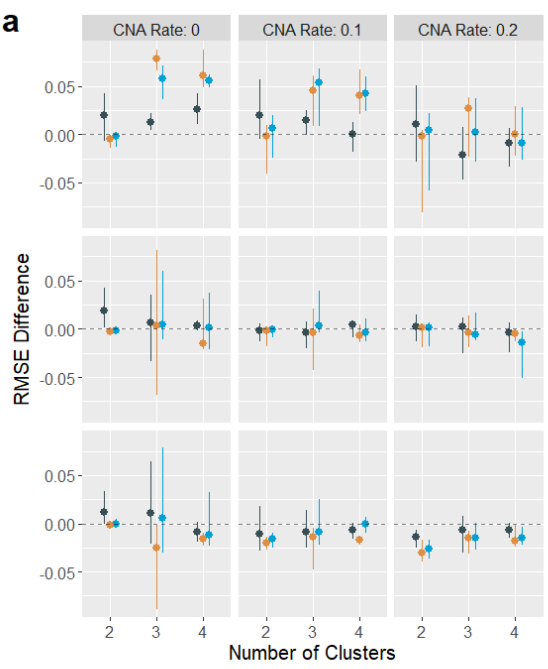

b

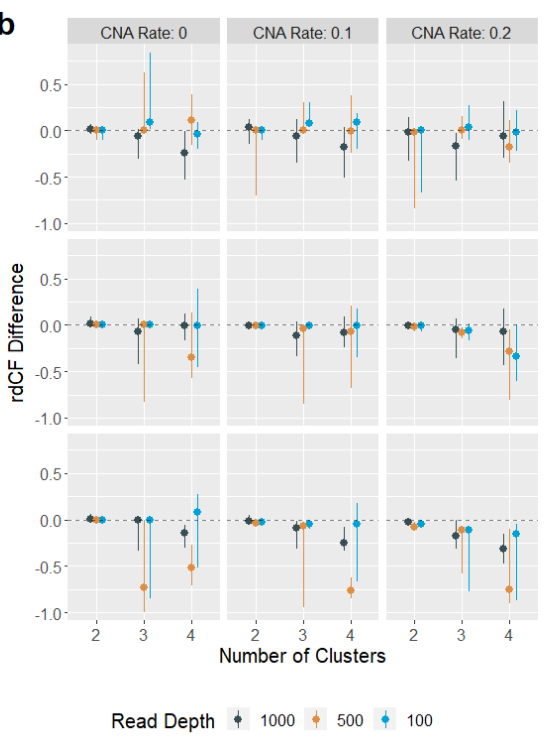

C

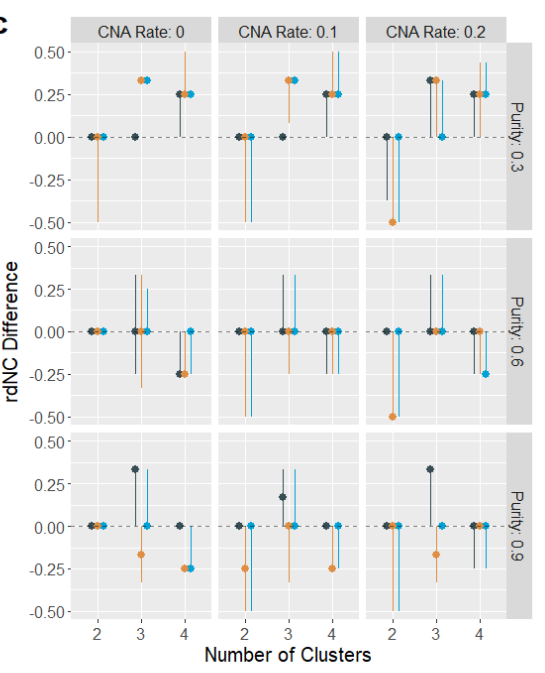

Supplementary Figure 1: Comparison between CliP and PhyloWGS in the simulation study. 


\section{Supplementary Text}

\subsection{Preliminaries}

The alternating direction method of multipliers (ADMM) is an algorithm that solves convex optimization problems by breaking them into smaller pieces. Therefore, to ensure the convexity of the likelihood (equation (6) in the main text), a three-part piece-wise linear approximation was used to approximate $g\left(\omega_{i}\right)$, which is defined in Equation (4) from the main text (see section 3 for details). Below is an example of such an approximation. We assume the linear approximation in CliP takes the form of $g\left(\omega_{i}\right) \approx u_{i}+v_{i} \omega_{i}$.

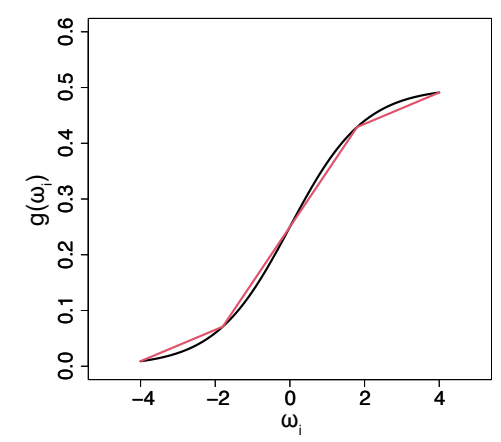

A three-part piece-wise linear approximation to approximate $g\left(\omega_{i}\right)$.

By the augmented Lagrangian method, we can obtain the estimation of parameters by minimizing

$$
L(\boldsymbol{\omega}, \boldsymbol{\eta}, \boldsymbol{\tau} ; \lambda)=S(\boldsymbol{\omega}, \boldsymbol{\eta} ; \lambda)+\frac{\alpha_{k}}{2} \sum_{i<j}\left(\omega_{i}-\omega_{j}-\eta_{i j}\right)^{2}-\sum_{i<j} \tau_{i j}\left(\omega_{i}-\omega_{j}-\eta_{i j}\right)
$$

where the dual variables $\boldsymbol{\tau}=\left\{\tau_{i j}, i<j\right\}$ are Lagrange multipliers, and $\alpha_{k}$ is the penalty parameter. It is noteworthy that when $\gamma>1 / \alpha_{k}+1$, the objective function $L(\boldsymbol{\omega}, \boldsymbol{\eta}, \boldsymbol{\tau} ; \lambda)$ with SCAD is convex with respect to $\eta_{i j}$ (induction see Section 3.2). Furthermore, the minimizer of $L(\boldsymbol{\omega}, \boldsymbol{\eta}, \boldsymbol{\tau} ; \lambda)$ with respect to $\eta_{i j}$ is unique and has a closed-form expression for given $(\boldsymbol{\omega}, \boldsymbol{\eta}, \boldsymbol{\tau})$.

For given $\left(\boldsymbol{\omega}, \alpha_{k}, \boldsymbol{\tau}\right), \hat{\eta}_{i j}$ is updated by solving

$$
\hat{\eta}_{i j}=\underset{\eta_{i j}}{\operatorname{argmin}} \frac{\alpha_{k}}{2}\left(\delta_{i j}-\eta_{i j}\right)^{2}+p_{\lambda}\left(\left|\eta_{i j}\right|\right)
$$

where $\delta_{i j}=\omega_{i}-\omega_{j}-\alpha_{k}^{-1} \tau_{i j}$. This is a one-dimensional SCAD-penalized linear regression problem which has a closed-from solution. Thus, the closed-form solution for SCAD penalty with $\gamma>$ $1 / \alpha_{k}+1$ is given by (Ma and Huang; 2017)

$$
\hat{\eta}_{i j}= \begin{cases}\mathrm{ST}\left(\delta_{i j}, \lambda / \alpha_{k}\right) & \text { if }\left|\delta_{i j}\right| \leq \lambda+\lambda / \alpha_{k} \\ \frac{S T\left(\delta_{i j}, \gamma \lambda /\left((\gamma-1) \alpha_{k}\right)\right)}{1-1 /\left((\gamma-1) \alpha_{k}\right)} & \text { if } \lambda+\lambda / \alpha_{k}<\left|\delta_{i j}\right| \leq \gamma \lambda \\ \delta_{i j} & \text { if }\left|\delta_{i j}\right|>\gamma \lambda\end{cases}
$$

where $\gamma$ is a tuning parameter that controls the concavity of the penalty (i.e., how rapidly the 
penalty tapers off), and $S T(x, t)$ is a soft thresholding operator, takes form:

$$
S T(x, t)= \begin{cases}\operatorname{sign}(x)(|x|-t) & \text { if }|x|>t \\ 0 & \text { if }|x|<=t\end{cases}
$$

\subsection{Computation}

To estimate $\boldsymbol{\omega}, \boldsymbol{\eta}, \boldsymbol{\tau}$, we designed an algorithm based on the ADMM for minimizing the objective function (1).

First, for a given $(\boldsymbol{\eta}, \boldsymbol{\tau})$, an update for $\boldsymbol{\omega}$ can be derived by setting $\partial L(\boldsymbol{\omega}, \boldsymbol{\eta}, \boldsymbol{\tau} ; \lambda) / \partial \boldsymbol{\omega}=0$. However, it is difficult to directly compute the derivative from the the likelihood function. Therefore, instead of using the theoretic variance, we could use the estimated variance at step $k$ to derive the estimator of $\boldsymbol{\omega}$ at step $k+1$. That is, we could derive the objective function (1) as follows:

$$
\begin{aligned}
L\left(\boldsymbol{\omega}, \boldsymbol{\eta}, \boldsymbol{\tau} ; \lambda, \boldsymbol{\omega}^{(k)}\right)= & S(\boldsymbol{\omega}, \boldsymbol{\eta} ; \lambda)+\frac{\alpha_{k}}{2} \sum_{i<j}\left(\omega_{i}-\omega_{j}-\eta_{i j}\right)^{2}-\sum_{i<j} \tau_{i j}\left(\omega_{i}-\omega_{j}-\eta_{i j}\right) \\
= & \frac{1}{2} \sum_{i=1}^{N} \frac{n_{i}\left(g\left(\omega_{i}\right)-\hat{\theta}_{i}\right)^{2}}{g\left(\omega_{i}\right)\left(1-g\left(\omega_{i}\right)\right)}+\sum_{1 \leq i<j \leq N} p_{\lambda}\left(\left|\eta_{i j}\right|\right) \\
& +\frac{\alpha_{k}}{2} \sum_{i<j}\left(\omega_{i}-\omega_{j}-\eta_{i j}\right)^{2}-\sum_{i<j} \tau_{i j}\left(\omega_{i}-\omega_{j}-\eta_{i j}\right) \\
= & \frac{1}{2} \sum_{i=1}^{N} \frac{n_{i}\left(g\left(\omega_{i}\right)-\hat{\theta}_{i}\right)^{2}}{g\left(\omega_{i}^{(k)}\right)\left(1-g\left(\omega_{i}^{(k)}\right)\right)}+\sum_{1 \leq i<j \leq N} p_{\lambda}\left(\left|\eta_{i j}\right|\right) \\
& +\frac{\alpha_{k}}{2} \sum_{i<j}\left(\omega_{i}-\omega_{j}-\eta_{i j}\right)^{2}-\sum_{i<j} \tau_{i j}\left(\omega_{i}-\omega_{j}-\eta_{i j}\right) \\
= & \frac{1}{2} \sum_{i=1}^{N} \frac{n_{i}\left(u_{i}+v_{i} \omega_{i}-r_{i} / n_{i}\right)^{2}}{g\left(\omega_{i}^{(k)}\right)\left(1-g\left(\omega_{i}^{(k)}\right)\right)}+\sum_{1 \leq i<j \leq N} p_{\lambda}\left(\left|\eta_{i j}\right|\right) \\
& +\frac{\alpha_{k}}{2} \sum_{i<j}\left(\omega_{i}-\omega_{j}-\eta_{i j}\right)^{2}-\sum_{i<j} \tau_{i j}\left(\omega_{i}-\omega_{j}-\eta_{i j}\right) \\
= & \frac{1}{2} \sum_{i=1}^{N}\left(A_{i}+B_{i} \omega_{i}\right)^{2}+\frac{\alpha_{k}}{2} \sum_{i<j}\left(\left(e_{i}-e_{j}\right)^{T} \boldsymbol{\omega}-\eta_{i j}-\alpha_{k}^{-1} \boldsymbol{\tau}\right)^{2}+C \\
= & \frac{1}{2}\|A+B \boldsymbol{\omega}\|^{2}+\frac{\alpha_{k}}{2}\left\|\Delta \boldsymbol{\omega}-\boldsymbol{\eta}-\boldsymbol{\alpha}_{\boldsymbol{k}}^{-1} \boldsymbol{\tau}\right\|^{2}+C
\end{aligned}
$$

where,

$$
A_{i}=\frac{\sqrt{n_{i}}\left(u_{i}-r_{i} / n_{i}\right)}{\sqrt{g\left(\omega_{i}^{(k)}\right)\left(1-g\left(\omega_{i}^{(k)}\right)\right)}}, \text { and } B_{i}=\frac{\sqrt{n_{i}}\left(v_{i}\right)}{\sqrt{g\left(\omega_{i}^{(k)}\right)\left(1-g\left(\omega_{i}^{(k)}\right)\right)}}
$$


$C$ is a function independent of $\boldsymbol{\omega}, e_{i}$ is $N \times 1$ vector whose $i$-th element is 1 and others are 0 , and $\Delta=\left\{\left(e_{i}-e_{j}\right), i<j\right\}^{T}, A=\left\{A_{1}, \ldots, A_{N}\right\}^{T}$, and

$$
B=\left[\begin{array}{cccc}
B_{1} & 0 & \cdots & 0 \\
0 & B_{2} & 0 \cdots & 0 \\
\cdots & \cdots & \ddots & 0 \\
0 & \cdots & 0 & B_{N}
\end{array}\right]
$$

Therefore,

$$
\boldsymbol{\omega}^{(k+1)}=\left(B^{T} B+\boldsymbol{\alpha}_{\boldsymbol{k}} \Delta^{T} \Delta\right)^{-1}\left[\boldsymbol{\alpha}_{\boldsymbol{k}} \Delta(\boldsymbol{\eta}-\boldsymbol{\tau})-B^{T} A\right]
$$

The direct inversion of matrix $B^{T} B+\boldsymbol{\alpha}_{\boldsymbol{k}} \Delta^{T} \Delta$ may not be feasible when $N$ is large, but fortunately we do not need to perform a $N \times N$ matrix inversion (Miller; 1981). Notice that $\Delta^{T} \Delta=N \mathbf{I}_{N}-$ $\mathbf{1}_{N} \mathbf{1}_{N}^{T}$, where $\mathbf{1}_{N}$ is a vector of length $N$ with all 1's; thus,

$$
B^{T} B+\boldsymbol{\alpha}_{\boldsymbol{k}} \Delta^{T} \Delta=\left(B^{T} B+N \boldsymbol{\alpha}_{\boldsymbol{k}} \boldsymbol{I}_{N}\right)-\boldsymbol{\alpha}_{\boldsymbol{k}} \mathbf{1}_{N} \mathbf{1}_{N}^{T}
$$

Now let $M=B^{T} B+N \boldsymbol{\alpha}_{\boldsymbol{k}} \boldsymbol{I}_{N}$, and $R=-\boldsymbol{\alpha}_{\boldsymbol{k}} \mathbf{1}_{N} \mathbf{1}_{N}^{T} . M$ is easy to invert since it is a diagonal matrix with non-zero diagonal elements, and $\operatorname{rank}(R)=1$. Then we have

$$
\left(B^{T} B+\boldsymbol{\alpha}_{\boldsymbol{k}} \Delta^{T} \Delta\right)^{-1}=(M+R)^{-1}=M^{-1}-\frac{1}{1+g} M^{-1} R M^{-1}
$$

where $g=\operatorname{trace}\left(R M^{-1}\right)$

Here we have avoided computing the inverse of a $N \times N$ matrix, instead, we only need to compute a series of matrix multiplications.

Lastly, $\tau$ is updated by a fixed schedule:

$$
\boldsymbol{\tau}^{(k+1)}=\boldsymbol{\tau}^{(k)}-\boldsymbol{\alpha}_{\boldsymbol{k}}\left(\Delta \boldsymbol{\omega}^{(k+1)}-\boldsymbol{\eta}^{(k+1)}\right)
$$

\section{Supplementary Induction}

\section{Proof of Equation (5) from the main paper}

Equation (5) is essentially a Gaussian approximation of Binomial distribution. From (Boas; 2006), we know that

$$
\begin{aligned}
f(x, n, p) & =\frac{n !}{x !(n-x) !} p^{x} q^{n-x} \\
& \sim \frac{1}{\sqrt{2 \pi n p q}} \exp \left\{-\frac{(x-n p)^{2}}{2 n p q}\right\}
\end{aligned}
$$

where $f$ follows a binomial distribution, $q=1-p$, and the sign $\sim$ means that the ratio tends to 1 as $n \rightarrow \infty$

Adapt equation (14) with our notation, where $n=n_{i}, p=\theta_{i}, q=1-\theta_{i}$, and $x=n_{i} \hat{\theta}_{i}$; $\hat{\theta}_{i}$ is what we have, while our target parameter is $\theta_{i}$ 
Then the likelihood is given by:

$$
\begin{aligned}
L & =\prod_{i=1}^{N} \frac{1}{\sqrt{2 \pi n p q}} \exp \left\{-\frac{(x-n p)^{2}}{2 n p q}\right\} \\
& =\prod_{i=1}^{N} \frac{1}{\sqrt{2 \pi n_{i} \theta_{i}\left(1-\theta_{i}\right)}} \exp \left\{-\frac{\left(n_{i} \hat{\theta}_{i}-n_{i} \theta_{i}\right)^{2}}{2 n \theta_{i}\left(1-\theta_{i}\right)}\right\}
\end{aligned}
$$

Then the loglikelihood is

$$
\begin{aligned}
l & =\log L \\
& =\log \left(\prod_{i=1}^{N} \frac{1}{\sqrt{2 \pi n_{i} \theta_{i}\left(1-\theta_{i}\right)}} \exp \left\{-\frac{\left(n_{i} \hat{\theta}_{i}-n_{i} \theta_{i}\right)^{2}}{2 n_{i} \theta_{i}\left(1-\theta_{i}\right)}\right\}\right) \\
& =\sum_{i=1}^{N} \log \left(\frac{1}{\sqrt{2 \pi n_{i} \theta_{i}\left(1-\theta_{i}\right)}}\right)-\frac{1}{2} \sum_{i=1}^{N} \frac{\left(n_{i} \hat{\theta}_{i}-n_{i} \theta_{i}\right)^{2}}{n_{i} \theta_{i}\left(1-\theta_{i}\right)} \\
& =-\frac{1}{2} \sum_{i=1}^{N} \frac{n_{i}\left(\hat{\theta}_{i}-\theta_{i}\right)^{2}}{\theta_{i}\left(1-\theta_{i}\right)}
\end{aligned}
$$

The first term of equation (19) is dropped because the problem is solved iteratively, i.e., $\theta_{i}\left(1-\theta_{i}\right)$ is known from $\theta_{i-1}\left(1-\theta_{i-1}\right)$.

\section{Brief induction of the Linear Approximation}

To ensure the concavity of the likelihood, we use a three-part piece-wise linear approximation to approximate $\theta_{i}=g\left(\omega_{i}\right)$

From the paper we have already have

$$
\begin{aligned}
\theta_{i} & =g\left(\omega_{i}\right) \\
& =\frac{b_{i}^{V} e^{\omega_{i}}}{\left(1+e^{\omega_{i}}\right)\left(2-2 \rho+c_{i}^{V} \rho\right)} \\
& =\frac{b_{i}^{V} e^{\omega_{i}}}{\left(1+e^{\omega_{i}}\right) A}
\end{aligned}
$$

where $A=2-2 \rho+c_{i}^{V} \rho$ and is a constant.

Let's set

$$
\begin{aligned}
& f(x)=\frac{b_{i}^{V} e^{\omega_{i}}}{\left(1+e^{\omega_{i}}\right) A} \\
& g(x)= \begin{cases}a_{1} x+b_{1}, & \text { when } x<x_{1} \\
a_{2} x+b_{2}, & \text { when } x \geq x_{2} \\
a_{3} x+b_{3}, & \text { when } x_{1} \leq x \leq x_{2}\end{cases}
\end{aligned}
$$


where $g(x)$ is used to approximate $f(x)$

Define $D_{n}$ as follows:

$$
D_{n}=\sup _{x}|f(x)-g(x)|
$$

To control the approximation error, we need to minimize $D_{n}$. In our application, we used a grid search to find the optimal separation points $x_{1}$ and $x_{2}$. Computationally, $D_{n}$ is estimated by

$$
D_{n}=\max _{x_{i}}\left|f\left(x_{i}\right)-g\left(x_{i}\right)\right|,
$$

Therefore, the search scheme is given by

$$
\operatorname{argmin}_{-M<x_{1}<x_{2}<M} D_{n}
$$




\section{References}

Boas, M. L. (2006). Mathematical methods in the physical sciences, John Wiley \& Sons.

Ma, S. and Huang, J. (2017). A concave pairwise fusion approach to subgroup analysis, Journal of the American Statistical Association 112(517): 410-423.

Miller, K. S. (1981). On the inverse of the sum of matrices, Mathematics Magazine 54(2): 67-72.

URL: http://www.jstor.org/stable/2690437 